\title{
The Orbital Stability of Solitary Wave Solutions for the Generalized Gardner Equation and the Influence Caused by the Interactions between Nonlinear Terms
}

\author{
Wei-Guo Zhang $\mathbb{D},{ }^{1}$ Xing-Qian Ling $\mathbb{D},{ }^{2}$ Xiang $\mathrm{Li}\left(\mathbb{D},{ }^{1}\right.$ and Shao-Wei $\mathrm{Li}^{3}$ \\ ${ }^{1}$ College of Science, University of Shanghai for Science and Technology, Shanghai 200093, China \\ ${ }^{2}$ Business School, University of Shanghai for Science and Technology, Shanghai 200093, China \\ ${ }^{3}$ School of Mathematics and Information Engineering, Taizhou University, Taizhou 317000, China \\ Correspondence should be addressed to Wei-Guo Zhang; zwgzwm@126.com
}

Received 16 April 2019; Accepted 21 May 2019; Published 20 June 2019

Academic Editor: Dimitri Volchenkov

Copyright (c) 2019 Wei-Guo Zhang et al. This is an open access article distributed under the Creative Commons Attribution License, which permits unrestricted use, distribution, and reproduction in any medium, provided the original work is properly cited.

In this paper, the orbital stability of solitary wave solutions for the generalized Gardner equation is investigated. Firstly, according to the theory of orbital stability of Grillakis-Shatah-Strauss, a general conclusion is given to determine the orbital stability of solitary wave solutions. Furthermore, on the basis of the two bell-shaped solitary wave solutions of the equation, the explicit expressions of the orbital stability discriminants are deduced to give the orbitally stable and instable intervals for the two solitary waves as the wave velocity changing. Moreover, the influence caused by the interaction between two nonlinear terms is also discussed. From the conclusion, it can be seen that the influences caused by this interaction are apparently when $0<p<4$, which shows the complexity of this system with two nonlinear terms. Finally, by deriving the orbital stability discriminant $d^{\prime \prime}(c)$ in the form of Gaussian hypergeometric function, the numerical simulations of several main conclusions are given in this paper.

\section{Introduction}

The Gardner equation originates from the study of the conservation law of KdV equation by Miura, Gardner, and Kruskal [1]. Because the Gardner equation possesses both quadratic and cubic term, many researchers recall the mixed KdV-MKdV equation,

$$
u_{t}+\beta u u_{x}+\gamma u^{2} u_{x}+u_{x x x}=0,
$$

as Gardner equation [2-5]. Equation (1) has important applications in many fields, such as nonlinear lattice, plasma physics, hydrodynamics, and solid state physics [6-9]. References [2-8] used different methods to solve the solitary wave solutions and some explicit solutions, such as the inverse scattering method and Hirota bilinear method [6, 7], the method of undetermined coefficients, and the extended tanh-function method $[2,3]$. Recently, $[10,11]$ studied the generalized Gardner equation,

$$
u_{t}+\left(\alpha+\beta u^{p}+\gamma u^{2 p}\right) u_{x}+u_{x x x}=0, \quad p>0,
$$

and used sub-ODE method and $G^{\prime} / G$ expansion method to solve the solitary wave solutions and other solutions of (2).

Equation (2) is the extension of the Gardner equation in [1]. When $p=1, \alpha=0$, (2) turns into (1). When $\gamma=0, \alpha=$ $0, \beta=1$, (2) turns into the generalized $\mathrm{KdV}$ equation,

$$
u_{t}+u^{p} u_{x}+u_{x x x}=0, \quad p>0 .
$$

This paper mainly studies the orbital stability of solitary wave solutions of generalized Gardner equation (2). The theory of the orbital stability of solitary wave solutions can be referred to in $[12,13]$. We found that the stability of solitary wave solutions studied by the mostly above literatures has only one nonlinear term in the equation. For example, for the stability of the solitary wave solutions for the generalized $\mathrm{KdV}$ equation (3) (it can be seen as the special case of the generalized Gardner equation (2)), many literatures have studied on it. By using variational method, [14-19] studied the stability of solitary wave solutions of the generalized $\mathrm{KdV}$ equation (3) and got the following conclusion: if $0<p<4$, the solitary wave solution of (3) is stable, and if $p>4$, 
it is instable. Meanwhile, [20] studied the orbital stability and instability of the solitary wave solutions of the nonlinear evolution equation which is much more complicated than (3) and obtained the following conclusion: if $0<p<4$, the solitary wave solution of (3) is orbitally stable, and if $p>4$, it is orbitally instable. Besides, $[21,22]$ obtained some sufficient conditions when the solutions of the equation,

$$
u_{t}+u^{p} u_{x}+u_{x x x}+\gamma u_{x x x x x}=0, \quad p=\text { const, } p>0,
$$

are stable, by using variational method and Lyapunov method, respectively. The reason why previous literature focused on the stability of solitary wave solutions for the evolution equations with only one nonlinear term may be that the analysis to the existence and other relative problems for the solitary wave solutions will become more complex and difficult when there are two or more than two nonlinear terms in the equation.

In this paper, based on our acknowledge on the solving the exact solitary wave solutions for nonlinear evolution equations, we give the exact solitary wave solutions of (2) with two nonlinear terms to directly state the existence. This reduces the high assumptions for the nonlinear parts of the equation when proving the existence of solitary wave solutions by analysis. Thus, it makes us apply the theory of orbital stability of Grillakis-Shatah-Strauss to the study of the orbital stability of solitary wave solutions for (2) possible.

The generalized Gardner equation (2) has another nonlinear term $\gamma u^{2 p} u_{x}$, comparing with the generalized $\mathrm{KdV}$ equation. So people want to know how about the orbital stability of the solitary wave solution of (2) naturally and the differences between the orbital stability of (2) and (3). In addition, there is only one bell-shaped solitary wave solution

$$
u(x-c t)=\left[\frac{c(p+1)(p+2)}{2} \operatorname{sech}^{2}\left(\frac{p \sqrt{c}}{2} \xi\right)\right]^{1 / p}
$$

in (3), but there are two solutions $u_{1}(x-c t)$ and $u_{2}(x-c t)$ in (2) (as shown in Lemma 1 below). Naturally, people want to know whether the orbital stability of the solutions $u_{1}(x-$ $c t)$ and $u_{2}(x-c t)$ is the same. In this paper, firstly by the application of the orbital stability theory of Grillakis-ShatahStrauss, we will get a general conclusion to judge the orbital stability of the generalized Gardner equation. Further, on the basis of the two exact bell-shaped solitary wave solutions of (2), we can deduce the explicit expressions of the orbital stability discriminants of two solutions and then give the intervals which make two solutions stable as the wave velocity changing. And we will also discuss the influences on the stable intervals caused by the two nonlinear terms in (2). The results in this paper show that, when the whole order of the nonlinear terms in (2) is high, just as when $p \geq 4$, the solitary wave solutions of (2) with two nonlinear terms and (3) with a single nonlinear term possess the same stability, both of which are instable. But when $0<p<4$, the orbital stability of (2) and (3) has apparent differences, which reflects the influences caused by two nonlinear terms of (2) on the orbital stability. Especially, in the case of $0<p<2, \beta<0$, we prove the following result: there exists $p_{0}\left(1<p_{0}<2\right)$; if $p \in\left(0, p_{0}\right)$, for any $c \in(\alpha,+\infty)$, the solitary wave solution $u_{1}(x-c t)$ is orbitally stable; if $p \in\left(p_{0}, 2\right)$, although the higher nonlinear term of (2) is less than 4 , there is still a part of wave velocity interval in $(\alpha,+\infty)$ making $u_{1}(x-c t)$ orbitally instable. The same conclusions of solitary wave solution $u_{2}(x-c t)$ can also be deduced in the situation of $0<p<2, \beta>0$. All the above conclusions indicate the complexity caused by the interaction between two nonlinear terms of (2).

To give explicit explanations of our conclusions, we use the transformation method to establish the orbital stability discriminant $d^{\prime \prime}(c)$ of the solitary wave solution in the form of Gauss hypergeometric function. Then the numerical simulating illustrations of several main conclusions can be got.

It is worth pointing out the discussion of the influence of the orbital stability of solitary wave solutions of the equation caused by the interactions between two nonlinear terms; the expression of orbital stability discriminant $d^{\prime \prime}(c)$ in the form of Gaussian hypergeometric function and giving the numerical simulations of the wave velocity intervals of the stability are original in this paper.

The method in this paper is an extension of GrillakisShatah-Strauss orbital stability theory. If an equation satisfies the conditions of Grillakis-Shatah-Strauss orbital stability theory and the solitary wave solutions of this equation can be solved, then the method in this paper can be referred to study the stability and the influence caused by the nonlinear terms.

Here we give some related results for the generalized Gardner equation as follows.

Lemma 1. Assume $c>\alpha$.

(1) If $\gamma>0$ or $\gamma \geq 0$ and $\beta>0$, then (2) has a bell-shaped solitary wave solution

$$
\begin{aligned}
u_{1}(x-c t) & =\left[\psi_{1}(\xi)\right]^{1 / p} \\
& =\left[\frac{A_{1} \operatorname{sech}^{2}\left(\tau_{1} / 2\right)(x-c t)}{2+B_{1} \operatorname{sech}^{2}\left(\tau_{1} / 2\right)(x-c t)}\right]^{1 / p},
\end{aligned}
$$

among which

$$
\begin{aligned}
& \tau_{1}=p \sqrt{c-\alpha} \\
& A_{1}=\frac{(c-\alpha)(p+1)(p+2) \sqrt{2 p+1}}{\sqrt{\beta^{2}(2 p+1)+\gamma(c-\alpha)(p+1)(p+2)^{2}}} \\
& B_{1}=-1+\frac{\beta A_{1}}{(c-\alpha)(p+1)(p+2)}
\end{aligned}
$$

(2) Let $p$ make $q^{1 / p}$ ( $q$ is negative) meaningful in $R$ (such as $p$ is odd), if $\gamma>0$, or $\gamma \geq 0$ and $\beta<0$, (2) also has bell-shaped solitary wave solution

$$
\begin{aligned}
u_{2}(x-c t) & =\left[\psi_{2}(\xi)\right]^{1 / p} \\
& =\left[\frac{A_{2} \operatorname{sech}^{2}\left(\tau_{2} / 2\right)(x-c t)}{2+B_{2} \operatorname{sech}^{2}\left(\tau_{2} / 2\right)(x-c t)}\right]^{1 / p}
\end{aligned}
$$


among which

$$
\begin{aligned}
\tau_{2} & =\tau_{1}, \\
A_{2} & =-A_{1}, \\
B_{2} & =-2-B_{1} .
\end{aligned}
$$

Remark 2. The above results can be deduced by the conclusion in $[10,23]$.

Remark 3. It is easy to examine that the function $\psi_{1}(\xi)$ in (6) is always positive under the condition of Lemma 1(1), and the function $\psi_{2}(\xi)$ in $(8)$ is always negative under the condition of Lemma 1(2).

\section{General Conclusions of the Orbital Stability of the Solitary Wave Solution for the Generalized Gardner Equation (2)}

The generalized Gardner equation (2) can be rewritten as the following Hamilton system:

$$
\frac{d u}{d t}=J E^{\prime}(u), \quad u \in X,
$$

where $X=H^{2}(R)$ with dual space $X^{*}=H^{-2}(R)$ and

$$
\begin{aligned}
& J=\frac{\partial}{\partial x}: D(J)\left(\subset X^{*}\right) \longrightarrow X, \\
& E(u)=\int_{R}\left[\frac{1}{2} u_{x}^{2}-\frac{\alpha}{2} u^{2}-\frac{\beta u^{p+2}}{(p+1)(p+2)}\right. \\
& \left.\quad-\frac{\gamma u^{2 p+2}}{(2 p+1)(2 p+2)}\right] d x, \\
& E^{\prime}(u)=-\alpha u-\frac{\beta u^{p+1}}{p+1}-\frac{\gamma u^{2 p+1}}{2 p+1}-u_{x x} .
\end{aligned}
$$

The inner product of $X$ is

$$
(f, g)=\int_{R}\left(f \cdot g+f^{\prime} \cdot g^{\prime}+f^{\prime \prime} \cdot g^{\prime \prime}\right) d x
$$

$$
\forall f, g \in X \text {. }
$$

And there is a natural isomorphism between $X$ and $X^{*}, I$ : $X \longrightarrow X^{*}$, satisfying $\langle I f, g\rangle=(f, g)$, where $\langle f, g\rangle=\int_{R} f$. $g d x, I=\partial^{4} / \partial x^{4}-\partial^{2} / \partial x^{2}+1$.

Let $T$ be a unitary operator group with a single parameter on $X$, satisfying

$$
T(s) u(\cdot)=u(\cdot-s), \quad \forall s \in R, u(\cdot) \in X ;
$$

obviously, $T^{\prime}(0)=-\partial / \partial x$ that

We can deduce $B=-1$ by $J B=T^{\prime}(0)$, so it can be defined

$$
\begin{aligned}
Q(u) & =\frac{1}{2}\langle B u, u\rangle=-\frac{1}{2} \int_{R} u^{2} d x, \\
Q^{\prime}(u) & =B u=-u, \\
Q^{\prime \prime}(u) & =-1 .
\end{aligned}
$$

From (15), we know solitary wave solutions (6), (8) of (2) in Lemma 1 can be rewritten as $T(c t) u_{i}(x)(i=1,2)$. Next, we consider the orbital stability of $T(c t) u_{i}(x)$. To avoid duplication, we denote $\phi_{c}(x)$ as one of $u_{1}(x)$ and $u_{2}(x)$.

Definition 4 (see [21]). The $\phi_{c}$-orbit $\left\{T(c t) \phi_{c}\right\}$ is stable if for all $\varepsilon<0$ there exists $\delta>0$ with the following property. If $\left\|u_{0}-\phi_{c}\right\|_{X}<\delta$ and $u(t)$ is a solution of (2) in some interval $\left[0, t_{0}\right)$ with $u(0)=u_{0}$, then $u(t)$ can be continued to a solution in $[0,+\infty)$ and

$$
\sup _{0<t<+\infty} \inf _{s \in R}\left\|u(t)-T(s) \phi_{c}\right\|_{X}<\varepsilon .
$$

Otherwise, the $\phi_{c}$-orbit is called unstable.

To prove the orbital stability of the solitary wave solution $T(c t) \phi_{c}$, we need to verify whether (2) and the solution $T(c t) \phi_{c}$ satisfy the three assumptions in [12].

Firstly, from Theorems 1 and 2 in [24], we can deduce the existence of solutions for the initial value problem of system (10).

Lemma 5 (see [19]). Let $s \geq 2$, for any fixed $u_{0} \in H^{s}(R)$, there exists a unique solution $u \in C\left([0, \infty) ; H^{s}(R)\right)$ in (2), which satisfies $u(0)=u_{0}$.

It is easy to show that $E(u), Q(u)$ which are defined in (12) and (16) satisfy

$$
\begin{aligned}
& E(u(t))=E(u(0))=E\left(u_{0}\right), \\
& Q(u(t))=Q(u(0))=Q\left(u_{0}\right) .
\end{aligned}
$$

Lemma 6. $\phi_{c}$ is the bounded solution of (2) and satisfies $E^{\prime}\left(\phi_{c}\right)-c Q^{\prime}\left(\phi_{c}\right)=0$.

Proof. By substituting $\phi_{c}$ into (2), we can obtain

$$
\alpha \phi_{c x}+\beta \phi_{c}^{p} \phi_{c x}+\gamma \phi_{c}^{2 p} \phi_{c x}+\phi_{c x x x}-c \phi_{c x}=0 .
$$

Integrating on both sides of (19), we can get

$$
\begin{aligned}
& -\frac{\beta \phi_{c}^{p+1}}{p+1}-\frac{\gamma \phi_{c}^{2 p+1}}{2 p+1}-\phi_{c x x}+(c-\alpha) \phi_{c}=C, \\
& C=\text { const. }
\end{aligned}
$$

And when $x \longrightarrow \infty, \phi_{c}, \phi_{c x}, \phi_{c x x} \longrightarrow 0$, then $C=0$; that is,

$$
\frac{\beta \phi_{c}^{p+1}}{p+1}-\frac{\gamma \phi_{c}^{2 p+1}}{2 p+1}-\phi_{c x x}+(c-\alpha) \phi_{c}=0 .
$$

So

$$
\begin{aligned}
E^{\prime}\left(\phi_{c}\right)-c Q^{\prime}\left(\phi_{c}\right)= & -\frac{\beta \phi_{c}^{p+1}}{p+1}-\frac{\gamma \phi_{c}^{2 p+1}}{2 p+1}-\phi_{c x x} \\
& +(c-\alpha) \phi_{c}=0 .
\end{aligned}
$$


Next we define operator $H_{c}: X \longrightarrow X^{*}, H_{c}=E^{\prime \prime}\left(\phi_{c}\right)-$ $c Q^{\prime \prime}\left(\phi_{c}\right)$, where

$$
\begin{aligned}
& E^{\prime \prime}(u)=-\alpha-\beta u^{p}-\gamma u^{2 p}-\partial_{x x}, \\
& Q^{\prime \prime}(u)=-1 .
\end{aligned}
$$

After calculating, we can get

$$
H_{c}=-\beta \phi_{c}^{p}-\gamma \phi_{c}^{2 p}-\partial_{x x}+c-\alpha, \quad c>\alpha .
$$

It is easy to know that $H_{c}$ is a self-conjugate operator, $H_{c}=$ $H_{c}^{*}$, which means $I^{-1} H_{c}$ is a bounded self-conjugate operator in $X$ and the eigenvalues of $H_{c}$ consist of the real number $\lambda$ such that $H_{c}-\lambda I$ irreversible.

From (19), we can know $\lambda=0$ is one eigenvalue of $H_{c}$,

$$
H_{c} T^{\prime}(0) \phi_{c}(x)=-H_{c} \phi_{c x}=0 .
$$

Let $Z^{\prime}=\left\{k \phi_{c x} \mid k \in R\right\}$, which contains the core $Z=\{u \in$ $\left.X \mid H_{c} u=0\right\}$ of $H_{c}$.

Now we are ready to prove the following lemma.

Lemma 7. For each $c \in(c-\alpha,+\infty), H_{c}$ has only one negative single eigenvalue. Its kernel space is spanned by $T^{\prime}(0) \phi_{c}$, and its other eigenvalues are positive, bounded, and far away from 0.

Proof. Since $x=0$ is the only zero of $\phi_{c x}$, we know 0 is the second eigenvalue of $H_{c}$ from Sturm-Liouville Theorem [25]. So $H_{c}$ only has one negative eigenvalue $-\sigma^{2}$, and its corresponding eigenfunction is denoted as $\chi$; then $H \chi=$ $-\sigma^{2} \chi,\langle\chi, \chi\rangle=1$.

For $H_{c}$, when $x \longrightarrow \infty, \phi_{c} \longrightarrow 0$, so $-\beta \phi_{c}^{p}-\gamma \phi_{c}^{2 p} \longrightarrow$ 0 . From Wely essential spectrum Theorem [26], the essential spectrum of $H_{c}$ satisfies ess $H_{c}=[c-\alpha,+\infty),(c>\alpha)$, so the desired result holds.

From the above discussion, we can make a spectral decomposition to $H_{c}$. Let

$$
\begin{aligned}
& Z=\left\{k_{1} \phi_{c x} \mid k_{1} \in R\right\}, \\
& N=\left\{k_{2} \chi \mid k_{2} \in R\right\}, \\
& P=\left\{p \in X \mid(p, \chi)=\left(p, \phi_{c x}\right)=0\right\} .
\end{aligned}
$$

For any $n(\neq 0) \in N,\left\langle H_{c} k_{2} \chi, k_{2} \chi\right\rangle=-k_{2}^{2} \sigma^{2}\langle\chi, \chi\rangle=$ $-k_{2}^{2} \sigma^{2}<0$, so $\left\langle H_{c} n, n\right\rangle<0$.

0 .

For any $z(\neq 0) \in Z,\left\langle H_{c} k_{1} \phi_{c x}, k_{1} \phi_{c x}\right\rangle=0$, so $\left\langle H_{c} z, z\right\rangle=$

For any $p(\neq 0) \in P$, according to Lemma 3.2 in [12], for any real function $p \in H^{1}(R)$ which satisfies $(p, \chi)=$ $\left(p, \phi_{c x}\right)=0$, there exists a positive $\beta>0$ making $\left\langle H_{c} p, p\right\rangle \geq$ $\beta\|p\|_{X}^{2}$, where $\beta$ is irrelevant to $p$; then $\left\langle H_{c} p, p\right\rangle>0$.

Therefore, space $X$ can be decomposed into direct sum $X=N \oplus Z \oplus P$, where $Z$ is the kernel space of $H_{c}, N$ is a finite-dimension space, and $P$ is a closed subspace.

We define $d(c): R \rightarrow R$ as $d(c)=E\left(\phi_{c}\right)-c Q\left(\phi_{c}\right)$. As a result of Lemmas 5-7, according to [12], we can get the following general conclusion of the solitary wave solution of (2).
Theorem 8. Let $u_{1}(x-c t), u_{2}(x-c t)$ be the solitary wave solutions of the generalized Gardner equation (2) given in Lemma 1, among which coefficients $p, \alpha, \beta, \gamma$ and wave velocity satisfy the hypothesis in Lemma 1 , respectively. Denote $\phi_{c}(c)$ as one of $u_{1}(x)$ and $u_{2}(x)$; then if $d^{\prime \prime}(c)>0, T(c t) \phi_{c}(x)$ is orbitally stable; else if $d^{\prime \prime}(c)<0, T(c t) \phi_{c}(x)$ is orbitally instable.

Remark 9. Since the skew-symmetric operator $J=\partial / \partial x$ : $D(J)\left(\subset X^{*}\right) \longrightarrow X$ is not onto, by directly using the conclusion in [20] or making similarly deduction, we can obtain the conclusion that if $d^{\prime \prime}(c)<0, T(c t) \phi_{c}(x)$ is orbitally instable in Theorem 8.

It can be inferred from Theorem 8 that we can examine the symbol of $d^{\prime \prime}(c)$ to study the orbital stability of the solitary wave solution of the generalized Gardner equation (2). Here

$$
\begin{aligned}
d^{\prime}(c) & =\left\langle E^{\prime}\left(\phi_{c}\right), \phi_{c}^{\prime}\right\rangle-c\left\langle Q^{\prime}\left(\phi_{c}\right), \phi_{c}^{\prime}\right\rangle-Q\left(\phi_{c}\right) \\
& =-Q\left(\phi_{c}\right)=\frac{1}{2} \int_{R} \phi_{c}^{2} d x .
\end{aligned}
$$

For convenience, we give the explicit expressions of the orbital stability discriminants of the solitary wave solution (6) and the solitary wave solution (8) of the generalized Gardner equation (2), respectively.

Firstly, we consider the orbital stability discriminant $d^{\prime \prime}(c)$ of the solitary wave solution (6) for (2). The solitary wave solution (6) can be rewritten as

$$
\begin{aligned}
u_{1}(x-c t) & =\left(\frac{A_{1} \operatorname{sech}^{2}\left(\tau_{1} / 2\right)(x-c t)}{2+B_{1} \operatorname{sech}^{2}\left(\tau_{1} / 2\right)(x-c t)}\right)^{1 / p} \\
& =\left(\frac{1}{d_{1}+d_{2} \cosh ^{2} d_{3}(x-c t)}\right)^{1 / p},
\end{aligned}
$$

among which

$$
\begin{aligned}
& d_{1}=\frac{B_{1}}{A_{1}} \\
& =\frac{-\sqrt{\beta^{2}(2 p+1)+\gamma(c-\alpha)(p+1)(p+2)^{2}}+\beta \sqrt{2 p+1}}{(c-\alpha)(p+1)(p+2) \sqrt{2 p+1}}
\end{aligned}
$$

$<0$

$d_{2}=\frac{4}{A_{1}}=\frac{2 \sqrt{\beta^{2}(2 p+1)+\gamma(c-\alpha)(p+1)(p+2)^{2}}}{(c-\alpha)(p+1)(p+2) \sqrt{2 p+1}}$

$>0$,

$d_{3}=\frac{\tau_{1}}{2}=\frac{p \sqrt{c-\alpha}}{2}>0$. 
In (27), we take $\phi_{c}(x)=u_{1}(x)$. Noting that $\cosh ^{2}\left(d_{3} x\right)$ is an even function, we have

$$
=\int_{0}^{+\infty}\left(\frac{1}{d_{1}+d_{2} \cosh ^{2}\left(d_{3} x\right)}\right)^{2 / p} d x
$$

$$
d^{\prime}(c)=\frac{1}{2} \int_{R}\left(\frac{1}{d_{1}+d_{2} \cosh ^{2}\left(d_{3} x\right)}\right)^{2 / p} d x
$$

Due to

$$
\begin{aligned}
\frac{d}{d c}\left(\frac{1}{d_{1}+d_{2} \cosh ^{2}\left(d_{3} x\right)}\right)^{2 / p} & =-\frac{2}{p} \frac{d_{1}^{\prime}+d_{2}^{\prime} \cosh ^{2}\left(d_{3} x\right)+2 d_{2} d_{3}^{\prime} x \cosh \left(d_{3} x\right) \sinh \left(d_{3} x\right)}{\left(d_{1}+d_{2} \cosh ^{2}\left(d_{3} x\right)\right)^{2 / p+1}} \\
& =-\frac{2}{p} \frac{\left(d_{1}^{\prime}-d_{1} d_{2}^{\prime} / d_{2}\right)+\left(d_{2}^{\prime} / d_{2}\right)\left(d_{1}+d_{2} \cosh ^{2}\left(d_{3} x\right)\right)+2 d_{2} d_{3}^{\prime} x \cosh \left(d_{3} x\right) \sinh \left(d_{3} x\right)}{\left(d_{1}+d_{2} \cosh ^{2}\left(d_{3} x\right)\right)^{2 / p+1}}
\end{aligned}
$$

and when $p>0$, for any fixed $c>\alpha$, each term on the right hand of formula (33) converges in $[0,+\infty)$,

$$
\begin{aligned}
& \int_{0}^{+\infty} \frac{x \cosh \left(d_{3} x\right) \sinh \left(d_{3} x\right)}{\left(d_{1}+d_{2} \cosh ^{2}\left(d_{3} x\right)\right)^{2 / p+1}} d x \\
& =\frac{1}{2 d_{2} d_{3}} \int_{0}^{+\infty} \frac{x d\left(d_{1}+d_{2} \cosh ^{2}\left(d_{3} x\right)\right)}{\left(d_{1}+d_{2} \cosh ^{2}\left(d_{3} x\right)\right)^{2 / p+1}} \\
& \quad=\frac{p}{4 d_{2} d_{3}} \int_{0}^{+\infty} \frac{1}{\left(d_{1}+d_{2} \cosh ^{2}\left(d_{3} x\right)\right)^{2 / p}} d x
\end{aligned}
$$

So when $p>0$, for any fixed $c>\alpha, \int_{0}^{+\infty}(d / d c)\left(1 /\left(d_{1}+\right.\right.$ $\left.\left.d_{2} \cosh ^{2}\left(d_{3} x\right)\right)\right)^{2 / p} d x$ converges.

For any real number $a, b>\alpha$, if wave velocity $c$ satisfies $\alpha<a<c<b, p \sqrt{a-\alpha} / 2<d_{3}<p \sqrt{b-\alpha} / 2$, and $\cosh ((p \sqrt{a-\alpha} / 2) x)<\cosh \left(d_{3} x\right)$ in $(0,+\infty)$, then there exists a constant $K$, independent of $c$, satisfying

$$
\begin{aligned}
0 & <\frac{1}{d_{1}+d_{2} \cosh ^{2}\left(d_{3} x\right)} \\
& <\frac{1}{d_{1}+d_{2} \cosh ^{2}((p \sqrt{a-\alpha} / 2) x)} \\
& =\frac{4 e^{p \sqrt{a-\alpha} x}}{e^{2 p \sqrt{a-\alpha} x}\left(1+2\left(2 d_{1} / d_{2}+1\right) e^{-p \sqrt{a-\alpha} x}+e^{-2 p \sqrt{a-\alpha} x}\right)} \\
& <\frac{4 K}{e^{p \sqrt{a-\alpha} x}} .
\end{aligned}
$$

If $c>\alpha, \int_{0}^{+\infty}(d / d c)\left(1 /\left(d_{1}+d_{2} \cosh ^{2}\left(d_{3} x\right)\right)\right)^{2 / p} d x$ not only converges, but also uniformly converges in any closed subintervals in $(\alpha,+\infty)$. Hence, according to (27), (33), and the derivation rule of the integral with parameter, we can deduce the orbital stability discriminant of the solitary wave solution (6) of (2),

$$
\begin{aligned}
d^{\prime \prime}(c) & =\int_{0}^{+\infty} \frac{d}{d c}\left(\frac{1}{d_{1}+d_{2} \cosh ^{2}\left(d_{3} x\right)}\right)^{2 / p} d x \\
& =\frac{2}{p} \int_{0}^{+\infty} \frac{\left(d_{1}^{\prime}-d_{1} d_{2}^{\prime} / d_{2}\right)+\left(d_{2}^{\prime} / d_{2}\right)\left(d_{1}+d_{2} \cosh ^{2}\left(d_{3} x\right)\right)+2 d_{2} d_{3}^{\prime} x \cosh \left(d_{3} x\right) \sinh \left(d_{3} x\right)}{\left(d_{1}+d_{2} \cosh ^{2}\left(d_{3} x\right)\right)^{2 / p+1}} d x \\
& =-\frac{2}{p}\left(\frac{d_{2}^{\prime}}{d_{2}}+\frac{d_{3}^{\prime} p}{2 d_{3}}\right) \int_{0}^{+\infty}\left(\frac{1}{d_{1}+d_{2} \cosh ^{2}\left(d_{3} x\right)}\right)^{2 / p} d x-\frac{2}{p}\left(d_{1}^{\prime}-\frac{d_{1} d_{2}^{\prime}}{d_{2}}\right) \int_{0}^{+\infty}\left(\frac{1}{d_{1}+d_{2} \cosh ^{2}\left(d_{3} x\right)}\right)^{2 / p+1} d x
\end{aligned}
$$

To avoid confusion, we denote the stability discriminant of the solitary wave solution (8) for the generalized Gardner equation (2) as $\bar{d}(c)$. By taking $\phi_{c}(x)=u_{2}(x)$ in (27), we have

$$
\bar{d}^{\prime}(c)=\frac{1}{2} \int_{R}\left(\frac{A_{2} \operatorname{sech}^{2}\left(\tau_{2} / 2\right) x}{2+B_{2} \operatorname{sech}^{2}\left(\tau_{2} / 2\right) x}\right)^{2 / p} d x
$$

$$
=\int_{0}^{+\infty}\left(\frac{1}{\bar{d}_{1}+\bar{d}_{2} \cosh ^{2}\left(\bar{d}_{3} x\right)}\right)^{2 / p} d x
$$

where $\bar{d}_{1}=B_{2} / A_{2}, \bar{d}_{2}=2 / A_{2}, \bar{d}_{3}=\tau_{2} / 2$. According to (8) and (9), we have 


$$
\begin{aligned}
& \bar{d}_{1}=d_{1}+d_{2}, \\
& \bar{d}_{2}=-d_{2}, \\
& \bar{d}_{3}=d_{3} .
\end{aligned}
$$

Similar to the discussion of the solitary wave solution (6), since $\int_{0}^{+\infty}(d / d c)\left(1 /\left(\bar{d}_{1}+\bar{d}_{2} \cosh ^{2}\left(\bar{d}_{3} x\right)\right)\right)^{2 / p} d x$ not only converges, but also uniformly converges in any closed subintervals in $(\alpha,+\infty)$ when $c>\alpha$, then according to (37) and the derivation rule of the integral with parameter, we have the orbital stability discriminant of the solitary wave solution (8),

$$
\begin{aligned}
\bar{d}^{\prime \prime}(c)= & \int_{0}^{+\infty} \frac{d}{d c}\left(\frac{1}{\bar{d}_{1}+\bar{d}_{2} \cosh ^{2}\left(\bar{d}_{3} x\right)}\right)^{2 / p} d x \\
= & -\frac{2}{p}\left(\frac{\bar{d}_{2}^{\prime}}{\bar{d}_{2}}+\frac{\bar{d}_{3}^{\prime} p}{2 \bar{d}_{3}}\right) \\
\cdot & \int_{0}^{+\infty}\left(\frac{1}{\bar{d}_{1}+\bar{d}_{2} \cosh ^{2}\left(\bar{d}_{3} x\right)}\right)^{2 / p} d x \\
- & \frac{2}{p}\left(\bar{d}_{1}^{\prime}-\frac{\bar{d}_{1} \bar{d}_{2}^{\prime}}{\bar{d}_{2}}\right) \\
& \int_{0}^{+\infty}\left(\frac{1}{\bar{d}_{1}+\bar{d}_{2} \cosh ^{2}\left(\bar{d}_{3} x\right)}\right)^{2 / p+1} d x .
\end{aligned}
$$

According to (38),

$$
\bar{d}_{1}^{\prime}-\frac{\bar{d}_{1} \bar{d}_{2}^{\prime}}{\bar{d}_{2}}=d_{1}^{\prime}-\frac{d_{1} d_{2}^{\prime}}{d_{2}}
$$

$$
\frac{\bar{d}_{2}^{\prime}}{\bar{d}_{2}}=\frac{d_{2}^{\prime}}{d_{2}}
$$

$$
\frac{\bar{d}_{3}^{\prime}}{\bar{d}_{3}}=\frac{d_{3}^{\prime}}{d_{3}} \text {. }
$$

By substituting (40) into (39), we can get

$$
\begin{gathered}
\bar{d}^{\prime \prime}(c)=\int_{0}^{+\infty} \frac{d}{d c}\left(\frac{1}{\bar{d}_{1}+\bar{d}_{2} \cosh ^{2}\left(\bar{d}_{3} x\right)}\right)^{2 / p} d x \\
=-\frac{2}{p}\left(\frac{d_{2}^{\prime}}{d_{2}}+\frac{d_{3}^{\prime} p}{2 d_{3}}\right) \\
\cdot \int_{0}^{+\infty}\left(\frac{1}{\bar{d}_{1}+\bar{d}_{2} \cosh ^{2}\left(\bar{d}_{3} x\right)}\right)^{2 / p} d x \\
-\frac{2}{p}\left(d_{1}^{\prime}-\frac{d_{1} d_{2}^{\prime}}{d_{2}}\right) \\
\cdot \int_{0}^{+\infty}\left(\frac{1}{\bar{d}_{1}+\bar{d}_{2} \cosh ^{2}\left(\bar{d}_{3} x\right)}\right)^{2 / p+1} d x .
\end{gathered}
$$

\section{The Orbital Stability of the Bell-Shaped Solitary Wave Solution of the Generalized Gardner Equation (2) When $p>2$}

\subsection{The Orbital Stability of the Solitary Wave Solution (6)}

When $p>2$

3.1.1. In the Case of $p>2, \gamma>0, \beta>0$. Note that the orbital stability discriminant of the solitary wave solution (6) of (2) is (36); we can get

$$
\begin{aligned}
d_{2}^{\prime} & =-\frac{2 \beta^{2}(2 p+1)+\gamma(c-\alpha)(p+1)(p+2)^{2}}{(c-\alpha)^{2}(p+1)(p+2) \sqrt{(2 p+1)\left(\beta^{2}(2 p+1)+\gamma(c-\alpha)(p+1)(p+2)^{2}\right)}}, \\
d_{1}^{\prime} & =-\frac{1}{2} d_{2}^{\prime}+\left(\frac{\beta}{(c-\alpha)(p+1)(p+2)}\right)^{\prime} \\
& =\frac{1}{(c-\alpha)^{2}(p+1)(p+2)}\left(\frac{2 \beta^{2}(2 p+1)+\gamma(c-\alpha)(p+1)(p+2)^{2}}{\sqrt{(2 p+1)\left(\beta^{2}(2 p+1)+\gamma(c-\alpha)(p+1)(p+2)^{2}\right)}}-\beta\right) \\
-\frac{2}{p}\left(d_{1}^{\prime}-\frac{d_{1} d_{2}^{\prime}}{d_{2}}\right) & =\frac{\beta \gamma(p+2)}{p(c-\alpha)\left(\beta^{2}(2 p+1)+\gamma(c-\alpha)(p+1)(p+2)^{2}\right)}
\end{aligned}
$$


From (43), we have that when $\beta>0, \gamma>0,-(2 / p)\left(d_{1}^{\prime}-\right.$ $\left.d_{1} d_{2}^{\prime} / d_{2}\right)>0$, and $0<\left(d_{1}+d_{2}\right) /\left(d_{1}+d_{2} \cosh ^{2}\left(d_{3} x\right)\right)<1(x \neq$ $0)$, then

$$
\begin{aligned}
& \int_{0}^{+\infty}\left(\frac{1}{d_{1}+d_{2} \cosh ^{2}\left(d_{3} x\right)}\right)^{2 / p+1} d x \\
& \quad<\frac{1}{d_{1}+d_{2}} \int_{0}^{+\infty}\left(\frac{1}{d_{1}+d_{2} \cosh ^{2}\left(d_{3} x\right)}\right)^{2 / p} d x .
\end{aligned}
$$

Substituting (43) and (44) into (36), we can get when $\beta>0$, $\gamma>0$

$$
d^{\prime \prime}(c)<\left(-\frac{d_{3}^{\prime}}{d_{3}}-\frac{2}{p} \frac{d_{1}^{\prime}+d_{2}^{\prime}}{d_{1}+d_{2}}\right)
$$

$$
\cdot \int_{0}^{+\infty}\left(\frac{1}{d_{1}+d_{2} \cosh ^{2}\left(d_{3} x\right)}\right)^{2 / p} d x
$$

In (45),

$$
\begin{aligned}
& \frac{d_{1}^{\prime}+d_{2}^{\prime}}{d_{1}+d_{2}} \\
& =-\frac{\beta \sqrt{2 p+1}+\sqrt{\beta^{2}(2 p+1)+\gamma(c-\alpha)(p+1)(p+2)^{2}}}{2(c-\alpha) \sqrt{\beta^{2}(2 p+1)+\gamma(c-\alpha)(p+1)(p+2)^{2}}}, \\
& \frac{d_{3}^{\prime}}{d_{3}}=\frac{1}{2(c-\alpha)}>0,
\end{aligned}
$$

so the coefficients in the right hand of (45) satisfy

$$
\begin{aligned}
-\frac{d_{3}^{\prime}}{d_{3}}-\frac{d_{1}^{\prime}+d_{2}^{\prime}}{d_{1}+d_{2}} & =\frac{1}{2(c-\alpha)}\left(\frac{2}{p} \frac{\beta \sqrt{2 p+1}+\sqrt{\beta^{2}(2 p+1)+\gamma(c-\alpha)(p+1)(p+2)^{2}}}{\sqrt{\beta^{2}(2 p+1)+\gamma(c-\alpha)(p+1)(p+2)^{2}}}-1\right) \\
& =\frac{1}{2 p(c-\alpha)}\left(\frac{-\beta^{2} p(p-4)(2 p+1)-\gamma(c-\alpha)(p+1)(p+2)^{2}(p-2)^{2}}{(2 \beta \sqrt{2 p+1}+(p-2) F(\alpha, \beta, \gamma, c, p)) F(\alpha, \beta, \gamma, c, p)}\right),
\end{aligned}
$$

where $\left.F(\alpha, \beta, \gamma, c, p)=\sqrt{\beta^{2}(2 p+1)+\gamma(c-\alpha)(p+1)(p+2)^{2}}\right)$.

We denote the numerator in the right hand of (47) as $G_{1}$, which means

$$
\begin{aligned}
G_{1}= & -\beta^{2} p(p-4)(2 p+1) \\
& -\gamma(c-\alpha)(p+1)(p+2)^{2}(p-2)^{2} .
\end{aligned}
$$

In (48), it can be shown that if $\gamma>0, c>\alpha, p \geq 4$, then $G_{1} \leq 0$, and $d^{\prime \prime}(c)<0$ follows from (45) and (47). When $2<p<4, G_{1} \leq 0$ is equivalent to

$$
c \geq \alpha+\frac{\beta^{2} p(4-p)(2 p+1)}{\gamma(p+1)(p+2)^{2}(p-2)^{2}} .
$$

So in the case of $\gamma>0, \beta>0, c>\alpha$, if $2<p<4$ and $c \geq \alpha+\beta^{2} p(4-p)(2 p+1) / \gamma(p+1)(p+2)^{2}(p-2)^{2}$, then $G_{1} \leq 0$, and $d^{\prime \prime}(c)<0$ follows from (45) and (47).

We can get following Proposition 10 from the above discussions.

Proposition 10. In the case of $\gamma>0, \beta>0, c>\alpha$, if $p \geq 4$, or $2<p<4$ and $c \geq \alpha+\beta^{2} p(4-p)(2 p+1) / \gamma(p+1)(p+2)^{2}(p-$ $2)^{2}$, the stability discriminant of the solitary wave solution (6) satisfies $d^{\prime \prime}(c)<0$.
3.1.2. In the Case of $\gamma>0, \beta<0, c>\alpha$. Since $\beta<0$, from (43), we have $-(2 / p)\left(d_{1}^{\prime}-d_{1} d_{2}^{\prime} / d_{2}\right)<0$, which implies that

$$
\begin{aligned}
- & \frac{2}{p}\left(d_{1}^{\prime}-\frac{d_{1} d_{2}^{\prime}}{d_{2}}\right) \\
& \cdot \int_{0}^{+\infty}\left(\frac{1}{d_{1}+d_{2} \cosh ^{2}\left(d_{3} x\right)}\right)^{2 / p+1} d x<0 .
\end{aligned}
$$

Therefore, according to (36), we can get when $\beta<0$,

$$
\begin{aligned}
& d^{\prime \prime}(c)<-\frac{2}{p}\left(\frac{d_{2}^{\prime}}{d_{2}}+\frac{d_{3}^{\prime} p}{2 d_{3}}\right) \\
& \quad \cdot \int_{0}^{+\infty}\left(\frac{1}{d_{1}+d_{2} \cosh ^{2}\left(d_{3} x\right)}\right)^{2 / p} d x<0 .
\end{aligned}
$$

After calculating, we can get that the coefficients in the right hand of (51) satisfy

$$
\begin{aligned}
& -\frac{2}{p}\left(\frac{d_{2}^{\prime}}{d_{2}}+\frac{d_{3}^{\prime} p}{2 d_{3}}\right) \\
& =\frac{\beta^{2}(2 p+1)(4-p)+\gamma(c-\alpha)(p+1)(p+2)^{2}(2-p)}{2 p(c-\alpha)\left(\beta^{2}(2 p+1)+\gamma(c-\alpha)(p+1)(p+2)^{2}\right)} .
\end{aligned}
$$

We use $G_{2}$ to denote the numerator of (52), which means

$$
\begin{aligned}
G_{2}= & \beta^{2}(2 p+1)(4-p) \\
& +\gamma(c-\alpha)(p+1)(p+2)^{2}(2-p) .
\end{aligned}
$$


From (53), we can obtain the following conclusion: if $\gamma>$ $0, c>\alpha, p \geq 4$, then $G_{2}<0$; if $2<p<4$ and $c \geq \alpha+\beta^{2}(2 p+$ $1)(4-p) / \gamma(p+1)(p+2)^{2}(p-2)$, then $G_{2} \leq 0$. So we can get the following Proposition 11 from (51).

Proposition 11. In the case of $\gamma>0, \beta<0, c>\alpha$, if $p \geq 4$, or $2<p<4$ and $c \geq \alpha+\beta^{2}(2 p+1)(4-p) / \gamma(p+1)(p+2)^{2}(p-$ $2)$, the stability discriminant of the solitary wave solution (6) satisfies $d^{\prime \prime}(c)<0$.

3.1.3. The Orbital Stability of the Solitary Wave Solution (6) in the Case of $2<p<4, \gamma>0, \beta>0$. From (43), we can get that $-(2 / p)\left(d_{1}^{\prime}-d_{1} d_{2}^{\prime} / d_{2}\right)>0$ if $\beta>0$. So we only need to consider which value $c$ takes; the first coefficient of (36) will be not less than 0 ; then we will have $d^{\prime \prime}(c)>0$. From (52) and (53), the first coefficient of (36) can be rewritten as

$$
\begin{aligned}
& -\frac{2}{p}\left(\frac{d_{2}^{\prime}}{d_{2}}+\frac{d_{3}^{\prime} p}{2 d_{3}}\right) \\
& =\frac{G_{2}}{2 p(c-\alpha)\left(\beta^{2}(2 p+1)+\gamma(c-\alpha)(p+1)(p+2)^{2}\right)} .
\end{aligned}
$$

From (53) and (54), we can obtain that, if $c$ satisfies

$$
c \leq \alpha+\frac{\beta^{2}(2 p+1)(4-p)}{\gamma(p+1)(p+2)^{2}(p-2)},
$$

then $G_{2} \geq 0$. So we can deduce $d^{\prime \prime}(c)>0$ from (36). And under this condition, we have Proposition 12.

Proposition 12. In the case of $2<p<4, \gamma>0, \beta>0$, if $c$ satisfies

$$
\alpha<c \leq \alpha+\frac{\beta^{2}(2 p+1)(4-p)}{\gamma(p+1)(p+2)^{2}(p-2)},
$$

then the stability discriminant of the solitary wave solution (6) satisfies $d^{\prime \prime}(c)>0$.

Due to

$$
\begin{gathered}
\frac{\beta^{2} p(2 p+1)(4-p)}{\gamma(p+1)(p+2)^{2}(p-2)^{2}} \\
>\frac{\beta^{2}(2 p+1)(4-p)}{\gamma(p+1)(p+2)^{2}(p-2)},
\end{gathered}
$$

we can get Theorem 13 from Propositions 10-12 and Theorem 8 .

Theorem 13. Assume $p>2, \gamma>0, c>\alpha$.

(1) If $p \geq 4$, or $2<p<4$ and $c \geq \alpha+\beta^{2} p(2 p+1)(4-$ $p) / \gamma(p+1)(p+2)^{2}(p-2)^{2}$, the solitary wave solution (6) is orbitally instable no matter $\beta>0$ or $\beta<0$.

(2) In the case of $\beta<0$, if $2<p<4$ and $c \geq \alpha+\beta^{2}(2 p+$ 1) $(4-p) / \gamma(p+1)(p+2)^{2}(p-2)$, the solitary wave solution (6) is orbitally instable.
(3) In the case of $2<p<4, \beta>0$, if $\alpha<c \leq \alpha+\beta^{2}(2 p+$ 1) $(4-p) / \gamma(p+1)(p+2)^{2}(p-2)$, the solitary wave solution (6) is orbitally stable.

\subsection{The Orbital Stability of the Solitary Wave Solution (8)}

When $p>2$

3.2.1. In the Case of $p>2, \gamma>0, \beta<0$. Note that $\bar{d}_{2}=$ $-d_{2}<0$, and $\cosh x \geq 1$ in $[0,+\infty)$; combining this with (38), we can deduce the following conclusion: no matter $\beta>0$ or $\beta<0, \bar{d}_{1}+\bar{d}_{2} \cosh ^{2}\left(\bar{d}_{3} x\right) \leq \bar{d}_{1}+\bar{d}_{2}=d_{1}<0$, which means $0<d_{1} /\left(\bar{d}_{1}+\bar{d}_{2} \cosh ^{2}\left(\bar{d}_{3} x\right)\right)<1(x \neq 0)$,

$$
\begin{aligned}
& \int_{0}^{+\infty}\left(\frac{1}{\bar{d}_{1}+\bar{d}_{2} \cosh ^{2}\left(\bar{d}_{3} x\right)}\right)^{2 / p} d x \\
& >\int_{0}^{+\infty}\left(\frac{1}{\bar{d}_{1}+\bar{d}_{2} \cosh ^{2}\left(\bar{d}_{3} x\right)}\right)^{2 / p} \frac{d_{1}}{\bar{d}_{1}+\bar{d}_{2} \cosh ^{2}\left(\bar{d}_{3} x\right)} d x .
\end{aligned}
$$

And from (43), we can deduce that $-\left(2 / p d_{1}\right)\left(d_{1}^{\prime}-d_{1} d_{2}^{\prime} /\right.$ $\left.d_{2}\right)>0$ when $\gamma>0, \beta<0$. So from (58), we have

$$
\begin{aligned}
& -\frac{2}{p d_{1}}\left(d_{1}^{\prime}-\frac{d_{1} d_{2}^{\prime}}{d_{2}}\right) \\
& \cdot \int_{0}^{+\infty}\left(\frac{1}{\bar{d}_{1}+\bar{d}_{2} \cosh ^{2}\left(\bar{d}_{3} x\right)}\right)^{2 / p} d x \\
& >-\frac{2}{p}\left(d_{1}^{\prime}-\frac{d_{1} d_{2}^{\prime}}{d_{2}}\right) \\
& \cdot \int_{0}^{+\infty}\left(\frac{1}{\bar{d}_{1}+\bar{d}_{2} \cosh ^{2}\left(\bar{d}_{3} x\right)}\right)^{2 / p+1} d x
\end{aligned}
$$

Then we substitute (59) into (41) and get

$$
\begin{aligned}
\bar{d}^{\prime \prime}(c)< & \left(-\frac{2}{p} \frac{d_{1}^{\prime}}{d_{1}}-\frac{d_{3}^{\prime}}{d_{3}}\right) \\
& \cdot \int_{0}^{+\infty}\left(\frac{1}{\bar{d}_{1}+\bar{d}_{2} \cosh ^{2}\left(\bar{d}_{3} x\right)}\right)^{2 / p} d x .
\end{aligned}
$$

Since

$$
\begin{aligned}
& \frac{d_{1}^{\prime}}{d_{1}} \\
& =\frac{\beta \sqrt{2 p+1}-\sqrt{\beta^{2}(2 p+1)+\gamma(c-\alpha)(p+1)(p+2)^{2}}}{2(c-\alpha) \sqrt{\beta^{2}(2 p+1)+\gamma(c-\alpha)(p+1)(p+2)^{2}}},
\end{aligned}
$$

then the coefficient in the right hand of (60) satisfies 


$$
\begin{aligned}
-\frac{2}{p} \frac{d_{1}^{\prime}}{d_{1}}-\frac{d_{3}^{\prime}}{d_{3}} & =\frac{1}{2(c-\alpha)}\left(\frac{2}{p} \frac{\sqrt{\beta^{2}(2 p+1)+\gamma(c-\alpha)(p+1)(p+2)^{2}}-\beta \sqrt{2 p+1}}{\sqrt{\beta^{2}(2 p+1)+\gamma(c-\alpha)(p+1)(p+2)^{2}}}-1\right) \\
& =-\frac{1}{2 p(c-\alpha)}\left(\frac{\beta^{2} p(2 p+1)(p-4)+\gamma(c-\alpha)(p+1)(p+2)^{2}(p-2)^{2}}{((p-2) F(\alpha, \beta, \gamma, c, p)-2 \beta \sqrt{2 p+1}) F(\alpha, \beta, \gamma, c, p)}\right),
\end{aligned}
$$

where $\left.F(\alpha, \beta, \gamma, c, p)=\sqrt{\beta^{2}(2 p+1)+\gamma(c-\alpha)(p+1)(p+2)^{2}}\right)$.

Here we set the numerator in the right hand of (62)

$$
\begin{aligned}
G_{3}= & \beta^{2} p(2 p+1)(p-4) \\
& +\gamma(c-\alpha)(p+1)(p+2)^{2}(p-2)^{2} .
\end{aligned}
$$

Since $\beta<0$, similar to (48) and (49), from (63), we can deduce that if $\gamma>0, c>\alpha, p \geq 4$, or $2<p<4$ and $c \geq$ $\alpha+\beta^{2} p(2 p+1)(4-p) / \gamma(p+1)(p+2)^{2}(p-2)^{2}$, then $G_{3} \geq 0$. And we can get Proposition 14 from (60) and (62).

Proposition 14. In the case of $\gamma>0, c>\alpha, \beta<0$, if $p \geq 4$, or $2<p<4$ and $c \geq \alpha+\beta^{2} p(2 p+1)(4-p) / \gamma(p+1)(p+2)^{2}(p-$ $2)^{2}$, the stability discriminant of the solitary wave solution (8) satisfies $\bar{d}^{\prime \prime}(c)<0$.

3.2.2. In the Case of $p>2, \gamma>0, \beta>0$. From (43), we can deduce that $-(2 / p)\left(d_{1}^{\prime}-d_{1} d_{2}^{\prime} / d_{2}\right)>0$ when $\beta>0$, $\int_{0}^{+\infty}\left(1 /\left(\bar{d}_{1}+\bar{d}_{2} \cosh ^{2}\left(\bar{d}_{3} x\right)\right)\right)^{2 / p+1} d x<0$. So from (41), we have

$$
\begin{aligned}
\bar{d}^{\prime \prime}(c)< & -\frac{2}{p}\left(\frac{d_{2}^{\prime}}{d_{2}}+\frac{d_{3}^{\prime} p}{2 d_{3}}\right) \\
& \cdot \int_{0}^{+\infty}\left(\frac{1}{\bar{d}_{1}+\bar{d}_{2} \cosh ^{2}\left(\bar{d}_{3} x\right)}\right)^{2 / p} d x .
\end{aligned}
$$

Then we can deduce the following proposition from (52) and (53).

Proposition 15. In the case of $\gamma>0, c>\alpha, \beta>0$, if $p \geq 4$, or $2<p<4$ and $c \geq \alpha+\beta^{2}(2 p+1)(4-p) / \gamma(p+1)(p+2)^{2}(p-$ $2)$, the stability discriminant of the solitary wave solution (8) satisfies $\bar{d}^{\prime \prime}(c)<0$.

3.2.3. The Orbital Stability of the Solitary Wave Solution (8) When $2<p<4, \gamma>0, \beta<0$. In the case of $\gamma>0, \beta<0$, we have $-(2 / p)\left(d_{1}^{\prime}-d_{1} d_{2}^{\prime} / d_{2}\right)<0, \bar{d}_{1}+\bar{d}_{2} \cosh ^{2}\left(\bar{d}_{3} x\right)<0$, so the second term of (41) is greater than 0 . And $\bar{d}^{\prime \prime}(c)>0$ only if the coefficient of the first term in (41) is greater than 0 . That is, $-(2 / p)\left(d_{2}^{\prime} / d_{2}+d_{3}^{\prime} p / 2 d_{3}\right) \geq 0$. And from the discussion in Section 3.1.3, we can deduce that if $\alpha<c \leq \alpha+\beta^{2}(2 p+1)(4-$ $p) / \gamma(p+1)(p+2)^{2}(p-2),-(2 / p)\left(d_{2}^{\prime} / d_{2}+d_{3}^{\prime} p / 2 d_{3}\right) \geq 0$, so $\bar{d}^{\prime \prime}(c)>0$, which means the solitary wave solution (8) is orbitally stable.
Combining with the above discussion and Propositions 14 and 15, we can get the following theorem.

Theorem 16. Assume $p>2, \gamma>0$, and set $p$ make $q^{1 / p}$ ( $q$ is negative) meaningful in $R$.

(1) If $p>4$, or $2<p<4$ and $c \geq \alpha+\beta^{2} p(2 p+1)(4-$ $p) / \gamma(p+1)(p+2)^{2}(p-2)^{2}$, the solitary wave solution (8) is orbitally instable no matter $\beta>0$ or $\beta<0$.

(2) In the case of $\beta>0$, if $2<p<4$ and $c \geq \alpha+\beta^{2}(2 p+$ $1)(4-p) / \gamma(p+1)(p+2)^{2}(p-2)$, the solitary wave solution (8) is orbitally instable.

(3) In the case of $2<p<4, \beta<0$, if $\alpha<c \leq \alpha+\beta^{2}(2 p+$ $1)(4-p) / \gamma(p+1)(p+2)^{2}(p-2)$, the solitary wave solution (8) is orbitally stable.

\section{The Orbital Stability of the Generalized Gardner Equation (2) When $0<p \leq 2$}

4.1. The Orbital Stability of the Solitary Wave Solution (6) of the Generalized Gardner Equation (2) When $0<p \leq 2$

4.1.1. The Simplification of the Orbital Stability Discriminant $d^{\prime \prime}(c)$ of the Solitary Wave Solution (6) When $0<p \leq 2$. Since $0<d_{1}+d_{2} \cosh ^{2}\left(d_{3} x\right) \longrightarrow \infty(x \longrightarrow \infty)$, in the case of $0<p \leq 2,\left(1 /\left(d_{1}+d_{2} \cosh ^{2}\left(d_{3} x\right)\right)\right)^{2 / p+1}$ converges to the bounded function which converges to 0 when $x \rightarrow \infty$ or constant 1 . So we can apply Mean Value Theorem of Integrals to the orbital stability discriminant (36) of the solitary wave solution (6). Then there exists $x^{*} \in R$, such that

$$
d^{\prime \prime}(c)=-\frac{2}{p}\left(\frac{1}{d_{1}+d_{2} \cosh ^{2}\left(d_{3} x^{*}\right)}\right)^{2 / p-1} \cdot I(c),
$$

where

$$
\begin{aligned}
I(c)= & \left(\frac{d_{2}^{\prime}}{d_{2}}+\frac{d_{3}^{\prime} p}{2 d_{3}}\right) \int_{0}^{+\infty} \frac{d x}{d_{1}+d_{2} \cosh ^{2}\left(d_{3} x\right)} \\
& +\left(d_{1}^{\prime}-\frac{d_{1} d_{2}^{\prime}}{d_{2}}\right) \int_{0}^{+\infty} \frac{d x}{\left(d_{1}+d_{2} \cosh ^{2}\left(d_{3} x\right)\right)^{2}} .
\end{aligned}
$$

Since $\cosh ^{2}\left(d_{3} x\right)=(1 / 2) \cosh \left(2 d_{3} x\right)+1 / 2$, then we have

$$
\begin{aligned}
& \int_{0}^{+\infty} \frac{d x}{\left(d_{1}+d_{2} \cosh ^{2}\left(d_{3} x\right)\right)^{2}} \\
& \quad=\frac{4}{d_{2}^{2}} \int_{0}^{+\infty} \frac{d x}{\left(\cosh \left(2 d_{3} x\right)+2\left(d_{1} / d_{2}\right)+1\right)^{2}} .
\end{aligned}
$$


By making the substitution $\cosh \left(2 d_{3} x\right)=z, z_{x}=2 d_{3} \sqrt{z^{2}-1}$ to the above formula, we can get

$$
\begin{gathered}
\int_{0}^{+\infty} \frac{d x}{\left(d_{1}+d_{2} \cosh ^{2}\left(d_{3} x\right)\right)^{2}}=\frac{2}{d_{2}^{2} d_{3}} \\
\cdot \int_{1}^{+\infty} \frac{d z}{\left(z+2\left(d_{1} / d_{2}\right)+1\right)^{2} \sqrt{z^{2}-1}} \\
=-\frac{1}{2 d_{1} d_{3}\left(d_{1}+d_{2}\right)} \\
-\frac{2 d_{1}+d_{2}}{4 d_{3}\left(-d_{1}\left(d_{1}+d_{2}\right)\right)^{3 / 2}}\left[\operatorname{sgn}\left(d_{2}\right) \frac{\pi}{2}\right. \\
\left.-\arctan \frac{2 d_{1}+d_{2}}{2 \sqrt{-d_{1}\left(d_{1}+d_{2}\right)}}\right] .
\end{gathered}
$$

By the same substitution, we can get

$$
\begin{gathered}
\int_{0}^{+\infty} \frac{d x}{d_{1}+d_{2} \cosh ^{2}\left(d_{3} x\right)}=\frac{1}{d_{2} d_{3}} \\
\cdot \int_{1}^{+\infty} \frac{d z}{\left(z+2\left(d_{1} / d_{2}\right)+1\right) \sqrt{z^{2}-1}} \\
=-\frac{1}{2 d_{3} \sqrt{-d_{1}\left(d_{1}+d_{2}\right)}}\left[\operatorname{sgn}\left(d_{2}\right) \frac{\pi}{2}\right. \\
\left.-\arctan \frac{2 d_{1}+d_{2}}{2 \sqrt{-d_{1}\left(d_{1}+d_{2}\right)}}\right] .
\end{gathered}
$$

By substituting (68) and (69) into (66), and noticing that $d_{2}>0$, then we have

$$
\begin{aligned}
& I(c)=-\frac{1}{2 d_{2}^{2} d_{3}}\left(d_{1}^{\prime}-\frac{d_{1} d_{2}^{\prime}}{d_{2}}\right) \frac{d_{2}^{2}}{d_{1}\left(d_{2}+d_{1}\right)} \\
& +\left[\left(\frac{d_{2}^{\prime}}{d_{2}}+\frac{d_{3}^{\prime} p}{2 d_{3}}\right)+\frac{2 d_{1}+d_{2}}{2 d_{1}\left(d_{2}+d_{1}\right)}\left(d_{1}^{\prime}-\frac{d_{1} d_{2}^{\prime}}{d_{2}}\right)\right] \\
& \cdot \frac{1}{d_{3} \sqrt{-4 d_{1}\left(d_{2}+d_{1}\right)}}\left[\frac{\pi}{2}\right. \\
& \left.-\arctan \frac{2 d_{1}+d_{2}}{2 \sqrt{-d_{1}\left(d_{1}+d_{2}\right)}}\right] .
\end{aligned}
$$

By simple calculating, we have

$$
\frac{d_{2}^{\prime}}{d_{2}}+\frac{d_{3}^{\prime} p}{2 d_{3}}
$$

$$
\begin{aligned}
& =-\frac{\beta^{2}(2 p+1)}{2(c-\alpha)\left(\beta^{2}(2 p+1)+\gamma(c-\alpha)(p+1)(p+2)^{2}\right)} \\
& \quad+\frac{1}{2(c-\alpha)}\left(\frac{p}{2}-1\right) \\
& \frac{2 d_{1}+d_{2}}{d_{1}\left(d_{1}+d_{2}\right)}=-\frac{2 \beta(2 p+1)}{\gamma(p+2)}, \\
& d_{3}\left(2 d_{1}+d_{2}\right)=\frac{\beta p}{\sqrt{c-\alpha}(p+1)(p+2)} \\
& d_{1}^{\prime}-\frac{d_{1} d_{2}^{\prime}}{d_{2}} \\
& =-\frac{\beta \gamma(p+2)}{2(c-\alpha)\left(\beta^{2}(2 p+1)+\gamma(c-\alpha)(p+1)(p+2)^{2}\right)} \\
& \frac{d_{2}^{2}}{d_{1}\left(d_{2}+d_{1}\right)} \\
& =-\frac{4\left(\beta^{2}(2 p+1)+\gamma(c-\alpha)(p+1)(p+2)^{2}\right)}{\gamma(c-\alpha)(p+1)(p+2)^{2}} .
\end{aligned}
$$

Then by substituting (71)-(75) into (70), we have

$$
\begin{aligned}
& I(c)=-\frac{\beta}{d_{2}^{2} d_{3}(p+1)(p+2)(c-\alpha)^{2}}-\left(1-\frac{p}{2}\right) \\
& . \frac{(p+1)(p+2)}{2 \beta p \sqrt{c-\alpha}} \frac{2 d_{1}+d_{2}}{\sqrt{-4 d_{1}\left(d_{2}+d_{1}\right)}}\left[\frac{\pi}{2}\right. \\
& \left.-\arctan \frac{2 d_{1}+d_{2}}{\sqrt{-4 d_{1}\left(d_{1}+d_{2}\right)}}\right] .
\end{aligned}
$$

Notice that

$$
\begin{aligned}
& M_{1}=\frac{2 d_{1}+d_{2}}{\sqrt{-4 d_{1}\left(d_{2}+d_{1}\right)}}\left[\frac{\pi}{2}\right. \\
& \left.-\arctan \frac{2 d_{1}+d_{2}}{\sqrt{-4 d_{1}\left(d_{1}+d_{2}\right)}}\right], \\
& k=\frac{\beta \sqrt{2 p+1}}{(p+2) \sqrt{\gamma(p+1)}},
\end{aligned}
$$

and then

$$
\begin{aligned}
\frac{2 d_{1}+d_{2}}{\sqrt{-4 d_{1}\left(d_{2}+d_{1}\right)}} & =\frac{\beta \sqrt{2 p+1}}{(p+2) \sqrt{\gamma(p+1)}} \cdot \frac{1}{\sqrt{c-\alpha}} \\
& =\frac{k}{\sqrt{c-\alpha}},
\end{aligned}
$$




$$
M_{1}=\frac{k}{\sqrt{c-\alpha}}\left[\frac{\pi}{2}-\arctan \frac{k}{\sqrt{c-\alpha}}\right]
$$

Substituting (77)-(79) into (76), then we have

$$
\begin{aligned}
I(c) & =-\frac{\beta(p+1)(p+2)(2 p+1)}{2 p \sqrt{c-\alpha}\left(\beta^{2}(2 p+1)+\gamma(c-\alpha)(p+1)(p+2)^{2}\right)} \\
& -\left(1-\frac{p}{2}\right) \frac{(p+1)(p+2)}{2 \beta p \sqrt{c-\alpha}} M_{1} \\
& =-\frac{(p+1)(p+2)}{2 p \sqrt{c-\alpha}}\left(\frac{\beta(2 p+1)}{\beta^{2}(2 p+1)+\gamma(c-\alpha)(p+1)(p+2)^{2}}\right. \\
& \left.+\frac{2-p}{2 \beta} M_{1}\right),
\end{aligned}
$$

so $(65)$ can be rewritten as

$$
\begin{aligned}
& d^{\prime \prime}(c)=\frac{(p+1)(p+2)}{\beta p^{2} \sqrt{c-\alpha}}\left(\frac{1}{d_{1}+d_{2} \cosh ^{2} d_{3} x^{*}}\right)^{2 / p-1} \\
& \cdot\left(\frac{\beta^{2}(2 p+1)}{\beta^{2}(2 p+1)+\gamma(c-\alpha)(p+1)(p+2)^{2}}\right. \\
& \left.+\frac{2-p}{2} M_{1}\right) .
\end{aligned}
$$

From Lemma 1, we know that $\psi_{1}(\xi)=1 /\left(d_{1}+\right.$ $\left.d_{2} \cosh ^{2} d_{3} x^{*}\right)$ is always positive in (81), and $d_{1}<0, d_{2}>0$, $d_{3}>0$ can be given by (29), (30), and (31), respectively.

\subsubsection{The Orbital Stability of the Solitary Wave Solution (6)}

(1) Case of $p=2$. From (81), we can get the following: if $\beta>0$, then $d^{\prime \prime}(c)>0$; if $\beta<0$, then $d^{\prime \prime}(c)<0$. So the solitary wave solution (6) is orbitally stable when $\beta>0$ and orbitally instable when $\beta<0$ in the case of $p=2$.

(2) Case of $0<p<2, \beta>0$. Firstly, we consider $M_{1}$ in (80) and (81). When $\beta>0$, we easily have $k>0, M_{1}>0$ from (77) and (79). Then we can get $d^{\prime \prime}(c)>0$ from (81), and the solitary wave solution (6) of (2) is orbitally stable.

(3) Case of $0<p<2, \beta<0$. From (77), it is easy to know $k<0$ when $\beta<0$. So $M_{1} \in(-\infty, 0)$ when $c \in(\alpha,+\infty)$. Now we substitute (77) into (81) and set $x=k / \sqrt{c-\alpha}$; then (81) can be rewritten as

$$
\begin{aligned}
& d^{\prime \prime}(c) \\
& \quad=\frac{(p+1)(p+2) k}{\beta p^{2}(c-\alpha)}\left(\frac{1}{d_{1}+d_{2} \cosh ^{2} d_{3} x^{*}}\right)^{2 / p-1} \\
& \quad \cdot\left(\frac{x}{x^{2}+1}+\frac{2-p}{2}\left[\frac{\pi}{2}-\arctan x\right]\right) .
\end{aligned}
$$

From (82), we can deduce that, in the case of $\beta<0$ (now $k<0), d^{\prime \prime}(c)>0$ is equivalent to $h_{1}(x)>0$. Here

$$
\begin{aligned}
& h_{1}(x)=\frac{x}{x^{2}+1}+\frac{2-p}{2}\left[\frac{\pi}{2}-\arctan x\right] \\
& x \in(-\infty, 0),
\end{aligned}
$$

and

$$
\begin{aligned}
& h_{1}^{\prime}(x)=\frac{1}{2\left(x^{2}+1\right)^{2}}\left((p-4) x^{2}+p\right), \\
& \\
& x \in(-\infty, 0) .
\end{aligned}
$$

From $h_{1}^{\prime}(x)=0$, we can get

$$
x_{0}=-\sqrt{\frac{p}{4-p}} .
$$

It is easy to obtain that if $x \in\left(-\infty, x_{0}\right)$, then $h_{1}^{\prime}(x)<0$, and if $x \in\left(x_{0}, 0\right)$, then $h_{1}^{\prime}(x)>0$. So $h_{1}(x)$ is monotone decreasing if $x \in\left(-\infty, x_{0}\right)$, monotone increasing if $x \in\left(x_{0}, 0\right)$, and $h_{1}(x)$ take the minimum value if $x=x_{0}$. And we have

$$
\begin{aligned}
& \lim _{x \rightarrow 0} h_{1}(x)=\lim _{x \rightarrow 0}\left(\frac{x}{x^{2}+1}+\frac{2-p}{2}\left[\frac{\pi}{2}-\arctan x\right]\right) \\
& \quad=\frac{2-p}{4} \pi, \\
& \lim _{x \rightarrow+\infty} h_{1}(x) \\
& =\lim _{x \rightarrow+\infty}\left(\frac{x}{x^{2}+1}+\frac{2-p}{2}\left[\frac{\pi}{2}-\arctan x\right]\right) \\
& =\frac{2-p}{2} \pi .
\end{aligned}
$$

So according to Intermediate Value Theorem of the continuous function, we can get the following proposition.

Proposition 17. Set $0<p<2, \beta<0$.

(1) If the minimum value $h_{1}\left(x_{0}\right)>0$, then $h_{1}(x)>0$ for any $x \in(-\infty, 0)$.

(2) If the minimum $h_{1}\left(x_{0}\right)<0$, then there exists $x_{1}, x_{2}$ (zero points of $\left.h_{1}(x)=0\right)$; if $x \in\left(-\infty, x_{1}\right)$ and $x \in\left(x_{2}, 0\right)$, then $h_{1}(x)>0$; if $x \in\left(x_{1}, x_{2}\right)$, then $h_{1}(x)<0$.

Since $x=k / \sqrt{c-\alpha}(k<0)$, according to Proposition 17 and (82) and (83), we have the following proposition.

Proposition 18. Set $0<p<2, \beta<0$.

(1) If minimum $h_{1}\left(x_{0}\right)<0$, then $d^{\prime \prime}(c)>0$ for any $c \in$ $(\alpha,+\infty)$; the solitary wave solution (6) of (2) is orbitally stable in $(\alpha,+\infty)$.

(2) If minimum $h_{1}\left(x_{0}\right)<0$, then there exists $x_{1}, x_{2} \in$ $(-\infty, 0)$ (the zero points of $\left.h_{1}(x)=0\right)$; if $c \in\left(\alpha, \alpha+k^{2} / x_{1}^{2}\right) \cup$ $\left(\alpha+k^{2} / x_{2}^{2},+\infty\right)$, then $d^{\prime \prime}(c)>0$; the solitary wave solution (6) is orbitally stable; if $c \in\left(\alpha+k^{2} / x_{1}^{2}, \alpha+k^{2} / x_{2}^{2}\right)$, then $d^{\prime \prime}(c)<0$; the solitary wave solution (6) is orbitally instable. 
Now substituting $x_{0}=-\sqrt{p /(4-p)}$ into $h(x)$, we can get the expression of $h_{1}\left(x_{0}\right)$ about $p$,

$$
\begin{aligned}
& h_{1}\left(x_{0}\right)=-\frac{\sqrt{p(4-p)}}{4} \\
&+\frac{2-p}{2}\left[\frac{\pi}{2}+\arctan \sqrt{\left.\frac{p}{4-p}\right],}\right. \\
& \\
& p \in(0,2) .
\end{aligned}
$$

Since $\lim _{p \longrightarrow 0} h_{1}\left(x_{0}\right)=\pi / 2, \lim _{p \longrightarrow 1} h_{1}\left(x_{0}\right)=(4 \pi-3 \sqrt{3}) /$ $12, \lim _{p \longrightarrow 2} h_{1}\left(x_{0}\right)=-1 / 2$, and $h_{1}\left(x_{0}\right)$ is monotone decreasing about $p$ in $(0,2)$; from Intermediate Value Theorem of the continuous function, we can deduce that there exists $p_{0} \in$ $(1,2)$ making the minimum $h_{1}\left(x_{0}\right)=0$ (from $(82)$; we can know $p_{0}$ also makes $\left.d^{\prime \prime}(c)=0\right)$. Then for any $p \in\left(0, p_{0}\right)$, the minimum value $h_{1}\left(x_{0}\right)>0$, while, for any $p \in\left(p_{0}, 2\right)$, the minimum value $h_{1}\left(x_{0}\right)<0$. Combining the above with Proposition 18, we can deduce the following proposition.

Proposition 19. We set $0<p<2, \beta<0$, and denote $p_{0}(1<$ $\left.p_{0}<2\right)$ as the zero point of $h_{1}\left(x_{0}\right)=0$ in (87), and then we have the following.

(1) When $p \in\left(0, p_{0}\right)$ (now $h_{1}\left(x_{0}\right)>0$ ), for any $c \in$ $(\alpha,+\infty), d^{\prime \prime}(c)>0$, the solitary wave solution (6) of equation (2) is orbitally stable in $(\alpha,+\infty)$.

(2) When $p \in\left(p_{0}, 2\right)$ (now $h_{1}\left(x_{0}\right)<0$ ), there exists $x_{1}(p), x_{2}(p)$ (the zero points of $\left.h_{1}(x)=0\right)$; if $c \in(\alpha, \alpha+$ $\left.k^{2} / x_{1}^{2}(p)\right) \cup\left(\alpha+k^{2} / x_{2}^{2}(p),+\infty\right)$, the solitary wave solution (6) is orbitally stable; if $c \in\left(\alpha+k^{2} / x_{1}^{2}(p), \alpha+k^{2} / x_{2}^{2}(p)\right)$, the solitary wave solution (6) is orbitally instable.

Now we set $c_{1}(p)=\alpha+k^{2} / x_{1}^{2}(p), c_{2}(p)=\alpha+k^{2} / x_{2}^{2}(p)$. Through the analysis, we can get the following theorem.

Theorem 20. Set $0<p \leq 2, \gamma>0, c\rangle \alpha$, for the solitary wave solution (6), we have the following conclusions.

(1) In the case of $p=2$, the solitary wave solution (6) is orbitally stable when $\beta>0$ and orbitally instable when $\beta<0$.

(2) In the case of $0<p<2, \beta>0$, the solitary wave solution (6) is orbitally stable for any $c \in(\alpha,+\infty)$.

(3) In the case of $0<p<2, \beta<0$, there exists $p_{0}(1<$ $\left.p_{0}<2\right)$ such that we have the following.

(1) If $p \in\left(0, p_{0}\right)$, for any $c \in(\alpha,+\infty)$, the solitary wave solution (6) is orbitally stable.

(2) When $p \in\left(p_{0}, 2\right)$, there exists $c_{1}(p), c_{2}(p)$; if $c \in$ $\left(\alpha, c_{1}(p)\right) \cup\left(c_{2}(p),+\infty\right)$, the solitary wave solution (6) is orbitally stable; if $c \in\left(c_{1}(p), c_{2}(p)\right)$, the solitary wave solution (6) is orbitally instable.

4.2. The Orbital Stability of the Solitary Wave Solution (8) of the Generalized Gardner Equation (2) When $0<p<2$

4.2.1. The Simplification of the Orbital Stability Discriminant $\bar{d}^{\prime \prime}(c)$ of the Solitary Wave Solution (8) When $0<p<2$. Since $\psi_{2}(\xi)$ is always negative in the solitary wave solution (8), we assume that $p$ makes $q^{1 / p}$ ( $q$ is negative) meaningful in $R$ (The case of $p=2$ is out of our discussion). In the case of $0<$ $p<2$, we make simplification for the discriminant $\bar{d}^{\prime \prime}(c)$ of the solitary wave solution (8). Similar to the discussion of the orbital stability of $u_{1}(x)$, by applying Mean Value Theorem of Integrals to (41), we can get that there exists $\bar{x} \in R$ such that

$$
\bar{d}^{\prime \prime}(c)<-\frac{2}{p}\left(\frac{1}{\bar{d}_{1}+\bar{d}_{2} \cosh ^{2}\left(\bar{d}_{3} x\right)}\right)^{2 / p-1} \cdot \bar{I}(c),
$$

$(\bar{x} \in R)$,

where

$\bar{I}(c)$

$$
\begin{aligned}
= & \left(\frac{d_{2}^{\prime}}{d_{2}}+\frac{d_{3}^{\prime} p}{2 d_{3}}\right) \int_{0}^{+\infty} \frac{d x}{\bar{d}_{1}+\bar{d}_{2} \cosh ^{2}\left(\bar{d}_{3} x\right)} \\
& +\left(d_{1}^{\prime}-\frac{d_{1} d_{2}^{\prime}}{d_{2}}\right) \int_{0}^{+\infty} \frac{d x}{\left(\bar{d}_{1}+\bar{d}_{2} \cosh ^{2}\left(\bar{d}_{3} x\right)\right)^{2}} .
\end{aligned}
$$

Since $\bar{d}_{i}$ and $d_{i}(i=1,2,3)$ satisfy (38) and (40), according to (71)-(75), we can obtain

$$
\begin{gathered}
\bar{I}(c)=-\frac{\beta}{d_{2}^{2} d_{3}(p+1)(p+2)}+\left(1-\frac{p}{2}\right) \\
. \frac{(p+1)(p+2)}{2 \beta p \sqrt{c-\alpha}} \frac{2 d_{1}+d_{2}}{\sqrt{-4 d_{1}\left(d_{2}+d_{1}\right)}}\left[\frac{\pi}{2}\right. \\
\left.+\arctan \frac{2 d_{1}+d_{2}}{\sqrt{-4 d_{1}\left(d_{1}+d_{2}\right)}}\right] .
\end{gathered}
$$

Due to (78) and (79), we set

$$
\begin{gathered}
M_{2}=\frac{2 d_{1}+d_{2}}{\sqrt{-4 d_{1}\left(d_{2}+d_{1}\right)}}\left[\frac{\pi}{2}\right. \\
\left.+\arctan \frac{2 d_{1}+d_{2}}{\sqrt{-4 d_{1}\left(d_{1}+d_{2}\right)}}\right],
\end{gathered}
$$

and then we have

$$
M_{2}=\frac{k}{\sqrt{c-\alpha}}\left[\frac{\pi}{2}+\arctan \frac{k}{\sqrt{c-\alpha}}\right] .
$$

So we have

$$
\begin{aligned}
& \bar{I}(c)=-\frac{\beta(p+1)(p+2)(2 p+1)}{2 p \sqrt{c-\alpha}\left(\beta^{2}(2 p+1)+\gamma(c-\alpha)(p+1)(p+2)^{2}\right)} \\
&+\left(1-\frac{p}{2}\right) \frac{(p+1)(p+2)}{2 \beta p \sqrt{c-\alpha}} M_{2}
\end{aligned}
$$




$$
\begin{aligned}
& =-\frac{(p+1)(p+2)}{2 \beta p \sqrt{c-\alpha}}\left(\frac{\beta^{2}(2 p+1)}{\beta^{2}(2 p+1)+\gamma(c-\alpha)(p+1)(p+2)^{2}}\right. \\
& \left.-\frac{2-p}{2} M_{2}\right), \\
\bar{d}^{\prime \prime}(c) & =\frac{(p+1)(p+2)}{\beta p^{2} \sqrt{c-\alpha}}\left(\frac{1}{\bar{d}_{1}+\bar{d}_{2} \cosh ^{2} \bar{d}_{3} \bar{x}^{*}}\right)^{2 / p-1} \\
& \cdot\left(\frac{\beta^{2}(2 p+1)}{\beta^{2}(2 p+1)+\gamma(c-\alpha)(p+1)(p+2)^{2}}-\frac{2-p}{2} M_{2}\right) .
\end{aligned}
$$

According to Lemma $1, \psi_{2}(\xi)=1 /\left(\bar{d}_{1}+\bar{d}_{2} \cosh ^{2} \bar{d}_{3} \bar{x}^{*}\right)$ in (94) is always negative. From the relationships between $d_{i}$ and $\bar{d}_{i}(i=1,2,3)$ in $(38)$, we can know $\bar{d}_{1}>0, \bar{d}_{2}<0, \bar{d}_{3}>0$.

\subsubsection{The Orbital Stability of the Solitary Wave Solution (8) When $0<p<2$}

(1) The Orbital Stability of the Solitary Wave Solution (8) When $0<p<2, \beta<0$. Firstly, from (77), it is easy to get $k<0$. Then we can substitute it into (92) and easily obtain $M_{2}<0$. Furthermore, $\bar{d}^{\prime \prime}(c)>0$ can be deduced from (94), so the solitary wave solution (8) of (2) is orbitally stable.

(2) The Orbital Stability of the Solitary Wave Solution (8) When $0<p<2, \beta>0$. Firstly, from (77) and (92), it is easy to know that, in the case of $\beta>0$, if $k>0$ and $c \in(\alpha,+\infty)$, $M_{2} \in(0,+\infty)$.

Similar to discussions in Section 4.1.2, now we substitute (77) into (94) and set $x=k / \sqrt{c-\alpha}$; then (94) can be rewritten as

$$
\begin{aligned}
\bar{d}^{\prime \prime} & (c) \\
& =\frac{(p+1)(p+2) k}{\beta p^{2} \sqrt{c-\alpha}}\left(\frac{1}{\bar{d}_{1}+\bar{d}_{2} \cosh ^{2} \bar{d}_{3} \bar{x}^{*}}\right)^{2 / p-1} \\
& \cdot\left(\frac{x}{x^{2}+1}-\frac{2-p}{2}\left[\frac{\pi}{2}+\arctan x\right]\right) .
\end{aligned}
$$

Since $\psi_{2}(\xi)=1 /\left(\bar{d}_{1}+\bar{d}_{2} \cosh ^{2} \bar{d}_{3} \bar{x}^{*}\right)$ is always negative, from (95), it is easy to get that $\bar{d}^{\prime \prime}(c)>0$ is equivalent to $h_{2}(x)<0$. Here

$$
\begin{aligned}
& h_{2}(x)=\frac{x}{x^{2}+1}-\frac{2-p}{2}\left[\frac{\pi}{2}+\arctan x\right], \\
& x \in(0,+\infty) .
\end{aligned}
$$

Since

$$
h_{2}^{\prime}(x)=\frac{1}{2\left(x^{2}+1\right)^{2}}\left((p-4) x^{2}+p\right),
$$

we have

$$
x \in(0,+\infty),
$$

$$
x_{0}=\sqrt{\frac{p}{4-p}}=-x_{0}
$$

from $h_{2}^{\prime}(x)=0$. So we can deduce that if $h_{2}^{\prime}(x)>0$, $x \in\left(0, \bar{x}_{0}\right)$, and if $x \in\left(\bar{x}_{0},+\infty\right), h_{2}^{\prime}(x)<0$. So $h_{2}(x)$ is monotone increasing if $x \in\left(0, \bar{x}_{0}\right)$ and monotone decreasing if $x \in\left(\bar{x}_{0},+\infty\right)$, and $h_{2}(x)$ takes the maximum value when $x=\bar{x}_{0}$; meanwhile,

$$
\begin{aligned}
\lim _{x \rightarrow 0} h_{2}(x)=\lim _{x \rightarrow 0}\left(\frac{x}{x^{2}+1}-\frac{2-p}{2}\left[\frac{\pi}{2}+\arctan x\right]\right) \\
=-\frac{2-p}{4} \pi, \\
\lim _{x \rightarrow+\infty} h_{2}(x) \\
=\lim _{x \rightarrow+\infty}\left(\frac{x}{x^{2}+1}-\frac{2-p}{2}\left[\frac{\pi}{2}+\arctan x\right]\right) \\
=-\frac{2-p}{2} \pi .
\end{aligned}
$$

On the basis of Intermediate Value Theorem of continuous function, we have Proposition 21 about $h_{2}\left(\bar{x}_{0}\right)$ and $h_{2}(x)$.

Proposition 21. (1) If the maximum $h_{2}\left(\bar{x}_{0}\right)<0$, then $h_{2}(x)<$ 0 for any $x \in(0,+\infty)$.

(2) If the maximum $h_{2}\left(\bar{x}_{0}\right)>0$, there exists $\bar{x}_{1}, \bar{x}_{2} ;$ if $x \in$ $\left(0, \bar{x}_{1}\right)$ and $x \in\left(\bar{x}_{2},+\infty\right)$, then $h_{2}(x)<0$; if $x \in\left(\bar{x}_{1}, \bar{x}_{2}\right)$, then $h_{2}(x)>0$.

Since $\beta>0, x=k / \sqrt{c-\alpha}(k>0)$; then we can deduce Proposition 22 from Proposition 21 and (95).

Proposition 22. Set $0<p<2, \beta>0$.

(1) If the maximum $h_{2}\left(\bar{x}_{0}\right)<0$, then, for any $c \in(\alpha,+\infty)$, $\bar{d}^{\prime \prime}(c)>0$, the solitary wave solution (8) of (2) is orbitally stable.

(2) If the maximum $h_{2}\left(\bar{x}_{0}\right)>0$, then there exist $\bar{x}_{1}, \bar{x}_{2} \in$ $(0,+\infty)$; if $c \in\left(\alpha, \alpha+k^{2} / \bar{x}_{2}^{2}\right) \cup\left(\alpha+k^{2} / \bar{x}_{1}^{2},+\infty\right), \bar{d}^{\prime \prime}(c)>0$, the solitary wave solution (8) is orbitally stable; if $c \in(\alpha+$ $\left.k^{2} / \bar{x}_{2}^{2}, \alpha+k^{2} / \bar{x}_{1}^{2}\right), \bar{d}^{\prime \prime}(c)<0$, the solitary wave solution (8) is orbitally instable.

Now substituting $\bar{x}_{0}=\sqrt{p /(4-p)}$ into $h_{2}(x)$, we can get the expression of $h_{2}\left(\bar{x}_{0}\right)$ as follows:

$$
\begin{aligned}
h_{2}\left(\bar{x}_{0}\right)= & \frac{\sqrt{p(4-p)}}{4} \\
& -\frac{2-p}{2}\left[\frac{\pi}{2}+\arctan \sqrt{\frac{p}{4-p}}\right],
\end{aligned}
$$

$$
p \in(0,2) \text {. }
$$

Since $\lim _{p \rightarrow 0} h_{2}\left(\bar{x}_{0}\right)=-\pi / 2, \lim _{p \rightarrow 1} h_{2}\left(\bar{x}_{0}\right)=(3 \sqrt{3}-$ $4 \pi) / 12, \lim _{p \rightarrow 2} h_{2}\left(\bar{x}_{0}\right)=1 / 2$, and $h_{2}\left(\bar{x}_{0}\right)$ is monotone increasing in $(0,2)$, from Intermediate Value Theorem of the continuous function, we can deduce, there exists $\bar{p}_{0} \in(1,2)$ making the maximum $h_{2}\left(\bar{x}_{0}\right)=0$ (from (95), we can know that $\bar{p}_{0}$ also makes $\left.\bar{d}^{\prime \prime}(c)=0\right)$. So for any $p \in\left(0, \bar{p}_{0}\right)$, maximum $h_{2}\left(\bar{x}_{0}\right)<0$, and for any $p \in\left(\bar{p}_{0}, 2\right)$, maximum 
$h_{2}\left(\bar{x}_{0}\right)>0$. Summarizing the above discussion, we can get the following proposition.

Proposition 23. Set $0<p<2, \beta>0, \bar{p}_{0}\left(1<\bar{p}_{0}<2\right)$ as the zero point of $h_{2}\left(\bar{x}_{0}\right)=0$.

(1) When $p \in\left(0, \bar{p}_{0}\right)\left(h_{2}\left(\bar{x}_{0}\right)<0\right)$, for any $c \in$ $(\alpha,+\infty), \bar{d}^{\prime \prime}(c)>0$, the solitary wave solution (8) of (2) is orbitally stable on $(0,+\infty)$.

(2) When $p \in\left(\bar{p}_{0}, 2\right)\left(h_{2}\left(\bar{x}_{0}\right)>0\right)$, there exist $\bar{x}_{1}(p), \bar{x}_{2}(p)$; if $c \in\left(\alpha, \alpha+k^{2} / \bar{x}_{2}^{2}(p)\right) \cup\left(\alpha+k^{2} / \bar{x}_{1}^{2}(p),+\infty\right)$, the solitary wave solution (8) of (2) is orbitally stable; if $c \in\left(\alpha+k^{2} / \bar{x}_{2}^{2}(p), \alpha+\right.$ $\left.k^{2} / \bar{x}_{1}^{2}(p)\right)$, the solitary wave solution (8) is orbitally instable.

We set $\bar{c}_{1}(p)=\alpha+k^{2} / \bar{x}_{2}^{2}(p), \bar{c}_{2}(p)=\alpha+k^{2} / \bar{x}_{1}^{2}(p)$. From the above analysis, we can get the following theorem.

Theorem 24. Set $p$ make $q^{1 / p}$ ( $q$ is negative) meaningful in $R$ and $0<p<2, \gamma>0, c\rangle \alpha$; then for the solitary wave solution (8); we have the following conclusions.

(1) In the case of $0<p<2, \beta<0$, for any $c \in(\alpha,+\infty)$, the solitary wave solution (8) is orbitally stable.

(2) In the case of $0<p<2, \beta>0$, there exists $\bar{p}_{0}(1<$ $\left.\bar{p}_{0}<2\right)$ with the following properties:

(1) When $p \in\left(0, \bar{p}_{0}\right)$, for any $c \in(\alpha,+\infty)$, the solitary wave solution (8) is orbitally stable.

(2) When $p \in\left(\bar{p}_{0}, 2\right)$, there exists $\bar{c}_{1}(p), \bar{c}_{2}(p)$, if $c \in$ $\left(\alpha, \bar{c}_{1}(p)\right) \cup\left(\bar{c}_{2}(p),+\infty\right)$, such that the solitary wave solution (8) is orbitally stable; if $c \in\left(\bar{c}_{1}(p), \bar{c}_{2}(p)\right)$, the solitary wave solution (8) is orbitally instable.

4.3. The Numerical Simulation of the Orbital Stability of the Solitary Wave Solution. For making the results in Sections 3, 4.1, and 4.2 more intuitive, we use MAPLE to give the numerical simulations. To avoid duplication and save the space, we only give the illustrations for the stability of the solitary wave solution (6) for (2) (others can be similarly explained). We start from the orbital stability discriminant (36) of the solitary wave solution (6) for (2).

We denote the first integral formula in (36) as $I_{1}$; i.e.,

$$
I_{1}=\int_{0}^{+\infty}\left(d_{1}+d_{2} \cosh ^{2}\left(d_{3} x\right)\right)^{-2 / p} d x .
$$

By using $\cosh ^{2}\left(d_{3} x\right)=\cosh \left(2 d_{3} x\right) / 2+1 / 2$ and setting $\sigma=$ $2 d_{1} / d_{2}+1, \tau=2 / p$, we can get

$$
I_{1}=\left(\frac{2}{d_{2}}\right)^{\tau} \int_{0}^{+\infty}\left[\cosh \left(2 d_{3} x\right)+\sigma\right]^{-\tau} d x .
$$

Then we set $\cosh \left(2 d_{3} x\right)=\omega$ and have

$$
I_{1}=\frac{1}{2 d_{3}}\left(\frac{2}{d_{2}}\right)^{\tau} \int_{1}^{+\infty} \frac{d \omega}{[\omega+\sigma]^{\tau} \sqrt{\omega^{2}-1}} .
$$

With the help of MAPLE, the integral in (103) can be expressed as

$$
I(\sigma, \tau)=\int_{1}^{+\infty} \frac{d \omega}{[\omega+\sigma]^{\tau} \sqrt{\omega^{2}-1}}
$$

$$
\begin{aligned}
& =\frac{1}{2^{2-\tau} \Gamma(\tau)}\left[\Gamma\left(\frac{\tau}{2}\right)^{2}\right. \\
& \text {. hypergeom }\left(\left[\frac{\tau}{2}, \frac{\tau}{2}\right],\left[\frac{1}{2}\right], \sigma^{2}\right) \\
& -2 \sigma \Gamma\left(\frac{\tau+1}{2}\right)^{2} \\
& \text {. hypergeom } \left.\left(\left[\frac{\tau+1}{2}, \frac{\tau+1}{2}\right],\left[\frac{3}{2}\right], \sigma^{2}\right)\right],
\end{aligned}
$$

when hypergeom is Guass generalized hypergeometric function.

Similarly, we set the second integral formula in (36) as $I_{2}$; i.e.,

$$
I_{2}=\int_{o}^{+\infty}\left(d_{1}+d_{2} \cosh ^{2}\left(d_{3} x\right)\right)^{-(2 / p+1)} d x
$$

With similar transformation, we have

$$
I_{2}=\frac{1}{2 d_{3}}\left(\frac{2}{d_{2}}\right)^{\tau+1} \cdot I(\sigma, \tau+1),
$$

where $I(\sigma, \tau+1)$ can be obtained from (104) by replacing $\tau+1$ with $\tau$.

By substituting $d_{1}, d_{2}, d_{3}$ in (29)-(31) and $I_{1}$ and $I_{2}$ into (36) and setting $L_{1}=\beta / \sqrt{p+1}(p+2), L_{2}=\gamma /(2 p+1)$, we have

$$
\begin{aligned}
d^{\prime \prime}(c)= & \frac{1}{p \sqrt{c-\alpha}}\left(\frac{(c-\alpha) \sqrt{p+1}}{\sqrt{L_{1}^{2}+(c-\alpha) L_{2}}}\right)^{2 / p} \\
& \cdot F(c, p),
\end{aligned}
$$

where

$$
\begin{aligned}
& F(c, p) \\
& =\left(\frac{2 L_{1}^{2}+(c-\alpha) L_{2}}{p(c-\alpha)\left(L_{1}^{2}+(c-\alpha) L_{2}\right)}-\frac{1}{2(c-\alpha)}\right) I(\sigma, \tau) \\
& \quad+\frac{L_{1} L_{2}}{p\left(L_{1}^{2}+(c-\alpha) L_{2}\right)^{3 / 2}} I(\sigma, \tau+1) .
\end{aligned}
$$

We can use (107) to make the numerical simulation of the orbital stability of the solitary wave solution (6) on the basis of the expression $d^{\prime \prime}(c)$ which is expressed by Guass generalized hypergeometric function.

4.3.1. The Numerical Simulation of the Orbital Stability of the Solitary Wave Solution (6) When $p>2, \gamma>0, \beta>0$. We set $\alpha=1, \beta=1, \gamma=3$ in (107) and get the 3D image of $d^{\prime \prime}(c)$ about $(c, p)$ shown in Figure 1, where dark blue surface is the image of $d^{\prime \prime}(c)$ and green flat surface is the horizontal plane of $z=0$.

From Figure 1, we can get that the 3D image of $d^{\prime \prime}(c)$ has both positive and negative area when $\alpha=1, \beta=1, \gamma=3$, 


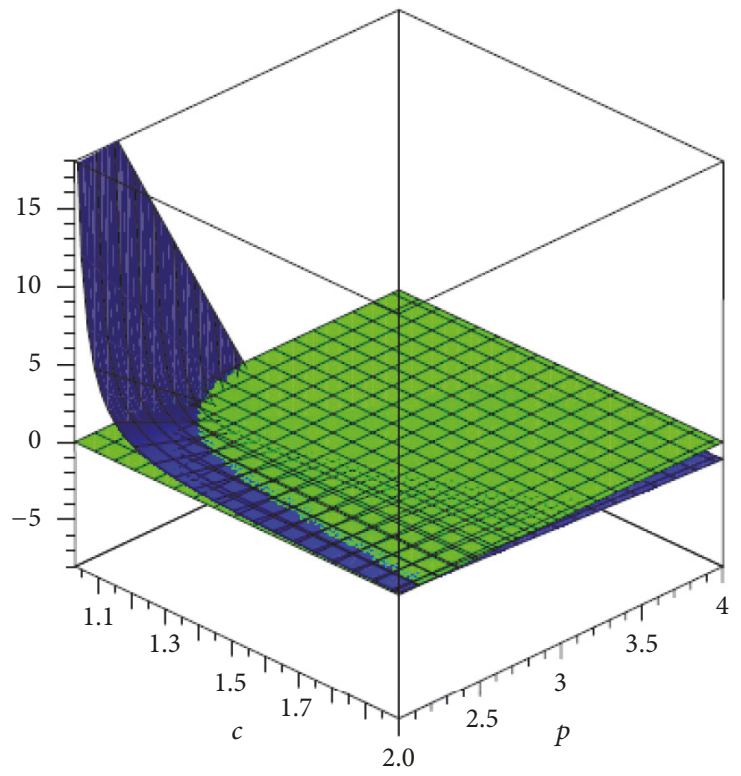

FIgUre 1: The 3D image of $d^{\prime \prime}(c)$ when $\alpha=1, \beta=1, \gamma=3,2<p<4$.

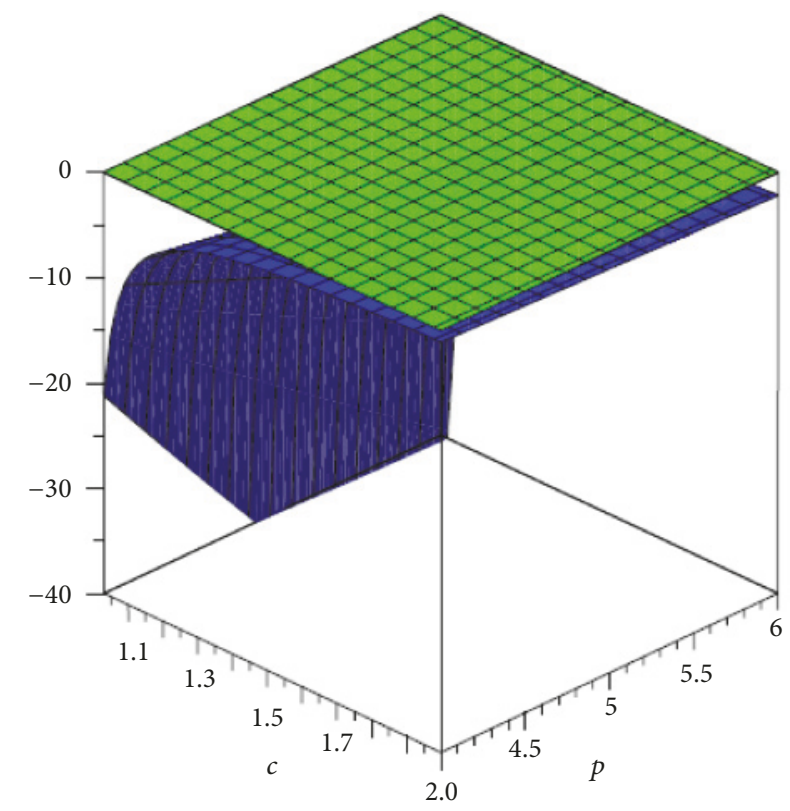

Figure 2: The 3D image of $d^{\prime \prime}(c)$ when $\alpha=1, \beta=1, \gamma=1, p>4$.

$2<p<4$, so there will be wave velocity interval that makes $d^{\prime \prime}(c)$ both positive and negative. From Figure 2, we can get that when $p>4$, the 3D image of $d^{\prime \prime}(c)$ is under the surface $z=0$. So $d^{\prime \prime}(c)$ is always negative when $p>4$.

In Figure 3 , the first curve is given by $c=\alpha+\beta^{2} p(2 p+$ $1)(4-p) / \gamma(p+1)(p+2)^{2}(p-2)^{2}$, the second curve is $d^{\prime \prime}(c)=0$, and the third curve is given by $c=\alpha+\beta^{2}(2 p+1)(4-$ $p) / \gamma(p+1)(p+2)^{2}(p-2)$. From Figure 3 , we can obtain that the area above the curve $d^{\prime \prime}(c)=0$ is the instable area, while

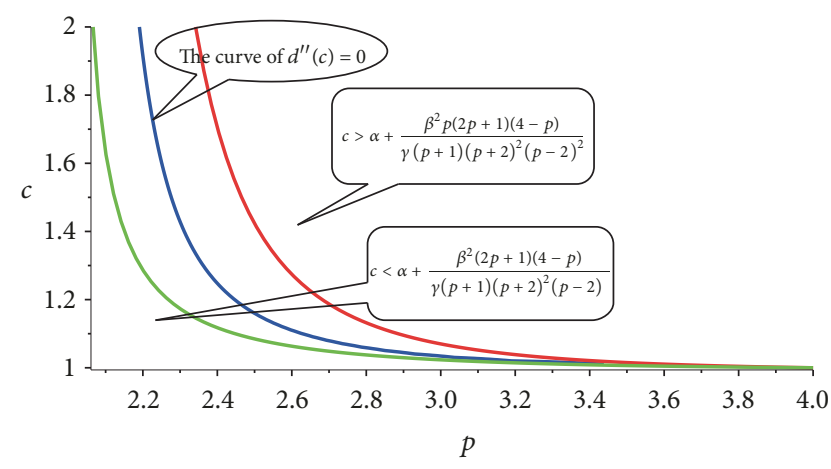

FIGURE 3: The stability region of the solitary wave solution (6) when $\alpha=1, \beta=1, \gamma=3,2<p<4$.

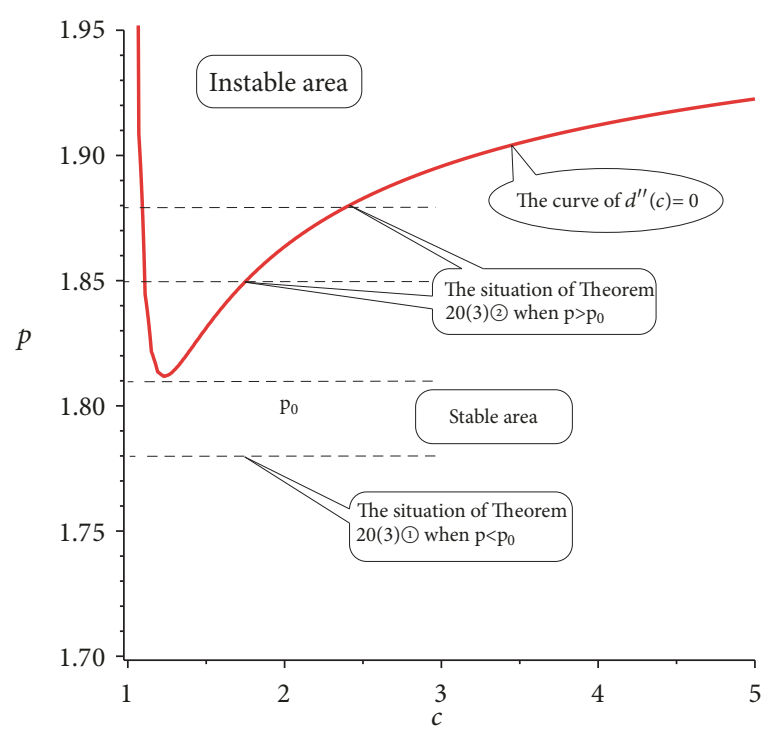

FIgURE 4: The curve of $d^{\prime \prime}(c)=0$ when $\alpha=1, \beta=-1, \gamma=3$, $0<p<2$.

the area below this curve is the stable area. But the instable area of the solitary wave solution (6) given by conclusion (1) of Theorem 13 lies above the first curve, and the stable region of the solitary wave solution (6) given by conclusion (3) lies below the third curve in Figure 3. The reason is we scale the inequalities in the analysis process, since it is hard to calculate the integral $\int_{0}^{+\infty}\left(d_{1}+d_{2} \cosh ^{2}\left(d_{3} x\right)\right)^{-2 / p} d x$ and $\int_{0}^{+\infty}\left(d_{1}+d_{2} \cosh ^{2}\left(d_{3} x\right)\right)^{-2 / p-1} d x$. So conditions of the stability and instability of the solitary wave solution (6) given in Theorem 13 when $2<p<4$ are only sufficient conditions.

4.3.2. The Numerical Simulation of the Orbital Stability of the Solitary Wave Solution (6) When $0<p<2, \gamma>0, \beta<0$. Using (107), by taking $\alpha=1, \beta=-1, \gamma=3,0<p<2$, we get the simulation results of $d^{\prime \prime}(c)=0$ which are shown in Figure 4. From Figure 4, we can see clearly that the curve $d^{\prime \prime}(c)=0$ takes the minimum point when $p=p_{0}$. If $p \epsilon$ $\left(0, p_{0}\right)$, the curve $d^{\prime \prime}(c)$ will fall into the area below the curve $d^{\prime \prime}(c)=0$. And if $p \in\left(p_{0}, 2\right)$, the curve $d^{\prime \prime}(c)$ will fall into the 


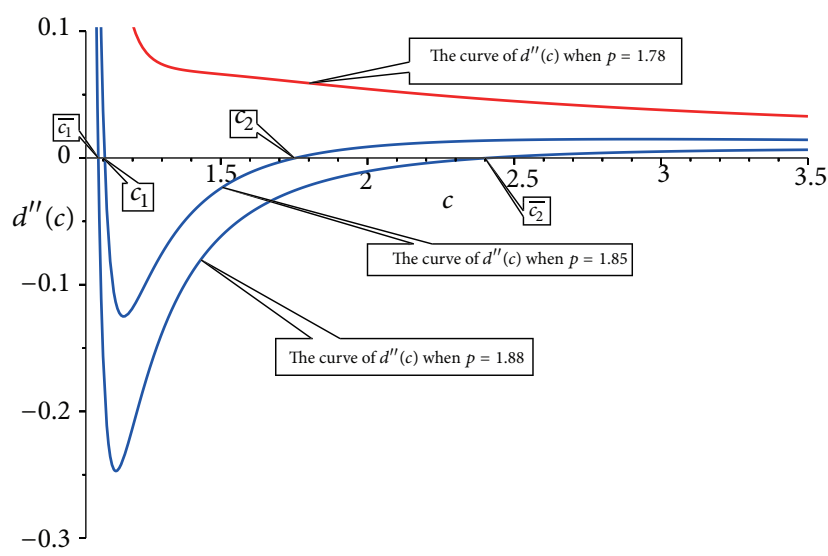

Figure 5: The curve image of $d^{\prime \prime}(c)$ when $p=1.78,1.85,1.88$.

area above the curve $d^{\prime \prime}(c)=0$. From Figure 4 , we can see that $p_{0} \approx 1.811$ under this case.

In the case of $\alpha=1, \beta=-1, \gamma=3$, when $p=1.78$, $p=1.85, p=1.88$, the curve $d^{\prime \prime}(c)$ is given in Figure 5. We can see that the curve $d^{\prime \prime}(c)$ is wholly above the straight line $z=0$ when $p=1.78$ (now $p<p_{0}$ ), which means $d^{\prime \prime}(c)>0$ for any $c \in(0,+\infty)$. The curve $d^{\prime \prime}(c)$ is above the straight line $z=0$ in $\left(0, c_{1}\right) \cup\left(c_{2},+\infty\right)$ and below the straight line $z=0$ in $\left(c_{1}, c_{2}\right)$ when $p=1.85$, which means, for any $c \in\left(0, c_{1}\right) \cup$ $\left(c_{2},+\infty\right), d^{\prime \prime}(c)>0$, while $d^{\prime \prime}(c)<0$ for any $c \in\left(c_{1}, c_{2}\right)$. Here $c_{1} \approx 1.1045454492, c_{2} \approx 1.7549789051$. The curve $d^{\prime \prime}(c)$ is above the straight line $z=0$ in $\left(0, \bar{c}_{1}\right) \cup\left(\bar{c}_{2},+\infty\right)$, and below the straight line $z=0$ in $\left(\bar{c}_{1}, \bar{c}_{2}\right)$ when $p=1.88$, which means $d^{\prime \prime}(c)>0$ for any $c \in\left(0, \bar{c}_{1}\right) \cup\left(\bar{c}_{2},+\infty\right)$ and $d^{\prime \prime}(c)<0$ for any $c \in\left(\bar{c}_{1}, \bar{c}_{2}\right)$. Here $c_{1} \approx 1.08300534093, c_{2} \approx 2.410645553$. In Figures 4 and 5 , it can be shown that simulation results prove the correctness of Conclusion 3 in Theorem 20.

\section{The Discussion of the Influence Caused by Interaction between Two Nonlinear Terms on the Stability of the Solitary Wave Solution}

In this paper, we study the orbital stability of the solitary wave solution of the generalized Gardner equation (2). And we obtain Theorems 13 and 20 for the solitary wave solution (6) and obtain Theorems 16 and 24 for the solitary wave solution (8).

Comparing with results that the solitary wave solution of the KdV equation (3) is orbitally instable when $p \geq 4$ and orbitally stable when $0<p<4$, it can be deduced that, when the order of the nonlinear terms in (3) and (2) is wholly high (in this paper $p \geq 4$ ), the solitary wave solutions of both equations are orbitally instable. However when $0<p<4$, the influence caused by interaction between two nonlinear terms on the orbital stability of the solitary wave solution for the generalized Gardner equation is obvious. Details are as follows.

(1) In the case of $2<p<4, \beta>0$, the order of the higher order nonlinear term in (2) is greater than 4 , and the effect of the lower order nonlinear term in (2) is obvious. According to Theorem 13, we can get that both orbitally stable and instable intervals exist for the solitary wave solution (6). If $\beta$ is larger, the interval which makes the solitary wave solution (6) stable is larger. If $\gamma$ is larger, the interval which makes the solitary wave solution (6) stable is smaller, and the interval that makes the solitary wave solution (6) instable is larger. This seems that the lower order nonlinear term $\beta u^{p} u_{x}$ plays a role in promoting stability in (2) when $\beta>0$.

(2) In the case of $\beta>0,0<p<2$, the order of the higher order nonlinear term in (2) is smaller than 4 . And both the solitary wave solution (6) of (2) and the solitary wave solution of (3) are orbitally stable. But if $p=2, \alpha=0$, from Theorem 20 in this paper, we can know that (2) can be rewritten as

$$
u_{t}+\beta u^{2} u_{x}+\gamma u^{4} u_{x}+u_{x x x}=0
$$

and its corresponding solitary wave solution (6) is orbitally stable for any $c \in(0,+\infty)$ when $\beta>0$. But according to [20], we can get that if we let $\beta=0$ in (109), then the solitary wave solution of the $\mathrm{KdV}$ equation

$$
u_{t}+\gamma u^{4} u_{x}+u_{x x x}=0
$$

is orbitally instable. By comparing, when $\beta>0$, the lower order nonlinear term in (109) seems to play a role in promoting the stability of the solitary wave solution (6).

(3) In the case of $0<p<2, \beta<0$, we consider the solitary wave solution (6). From Theorem 20, we can get that there exists $p_{0}\left(1<p_{0}<2\right)$, if $0<p<p_{0}$, the solitary wave solution (6) is orbitally stable in $(\alpha,+\infty)$, but if $p_{0}<p<2$, although the order of the higher order nonlinear term in (2) is less than 4 , there is still part of wave velocity interval $(\alpha,+\infty)$ in which the solution is orbitally instable. It seems that, for the solitary wave solution (6), when $\beta<0$, the term $\beta u^{p} u_{x}$ in (2) plays a role against the stability in equation.

(4) For the solitary wave solution (8) of (2), we can make the similar analysis as conclusions (1)-(3). From Theorems 16 and 24 , we can get that, for the solitary wave solution (8), the term $\beta u^{p} u_{x}$ in (2) plays a role promoting the stability in the equation if $\beta<0$ and plays a role against the stability in equation if $\beta>0$.

The method in this paper is an extension of the theory of orbital stability of Grillakis-Shatah-Strauss. The most fundamental result is that we find and prove, $\int_{0}^{+\infty}(d / d c)\left(1 /\left(d_{1}+\right.\right.$ $\left.\left.d_{2} \cosh ^{2}\left(d_{3} x\right)\right)\right)^{2 / p} d x$ converges for any fixed $c$ when $p>$ 0 and uniformly converges in any subintervals $[a, b]$ in $(\alpha,+\infty)$. Then we apply our method to calculate the integral with a parameter to establish the explicit expressions (36) and (41) of the orbital stability discriminant of the solitary wave solutions (6) and (8), respectively. All conclusions in this paper are obtained on the basis of the above two formulas and by the analysis method.

From the study in this paper, we can find that if an equation satisfies the conditions of the theory of orbital stability of Grillakis-Shatah-Strauss and the solitary wave solutions of equation can be solved, then the method in this paper can be referred to study the stability and instability of its 
solitary wave solutions. Since the exact explicit solitary wave solutions of a large number of soliton equations have been obtained in the field of Mathematics and Physics since 1980s, in order to further study the stability of these solitary wave solutions with explicit expressions, we think the method in this paper is worth being referred.

\section{Data Availability}

The data used to support the findings of this study are included within this article.

\section{Conflicts of Interest}

The authors declare that there are no conflicts of interest regarding the publication of this article.

\section{Acknowledgments}

This work was supported by National Nature Science Foundation of China [Grant no. 11471215].

\section{References}

[1] R. M. Miura, C. S. Gardner, and M. D. Kruskal, "Kortewegde Vries equation and generalizations: II. Existence of conservation laws and constants of motion," Journal of Mathematical Physics, vol. 9, pp. 1204-1209, 1968.

[2] Z. Fu, S. Liu, and S. Liu, "New kinds of solutions to Gardner equation," Chaos, Solitons \& Fractals, vol. 20, no. 2, pp. 301-309, 2004.

[3] A.-M. Wazwaz, "New solitons and kink solutions for the Gardner equation," Communications in Nonlinear Science and Numerical Simulation, vol. 12, no. 8, pp. 1395-1404, 2007.

[4] F. Q. Dou, J. A. Sun, K. P. Lv, G. H. Zhu, and X. R. Hong, "Selfsimilar solutions for the Gardner equation," Ziran Kexue Ban. Journal of Northwest Normal University, vol. 40, no. 1, pp. 35-37, 2004 (Chinese).

[5] Y. N. Tang, W. Xu, and J. W. Shen, "Solitary wave solutions to the Gardner equation," Chinese Journal of Engineering Mathematics, vol. 24, no. 1, pp. 119-127, 2007.

[6] W. Miki, "Wave propagation in nonlinear lattice I," Journal of the Physical Society of Japan, vol. 38, no. 3, pp. 673-680, 1975.

[7] M. Wadati, "Wave Propagation in Nonlinear Lattice. II," Journal of the Physical Society of Japan, vol. 38, no. 3, pp. 681-686, 1975.

[8] M. Toda, "Waves in nonlinear lattice," Progress of Theoretical and Experimental Physics Supplement, vol. 45, pp. 174-200, 1970.

[9] D. Shi-qiang, "Solitary waves at the interface of a two-layer fluid," Applied Mathematics and Mechanics-English Edition, vol. 3, no. 6, pp. 777-788, 1982 (Chinese).

[10] X. Z. Li and M. L. Wang, "A sub-ODE method for finding exact solutions of a generalized KDV-mKDV Equation with highorder nonlinear terms," Physics Letters A, vol. 361, no. 1-2, pp. 115-118, 2007.

[11] Q. Feng and B. Zheng, "Exact traveling wave solution for the general Gardner equation," in Proceedings of the American Conference on Applied Mathematics World Scientific and Engineering Academy and Society (WSEA), pp. 421-423, 2010.
[12] M. Grillakis, J. Shatah, and W. Strauss, "Stability theory of solitary waves in the presence of symmetry I," Journal of Functional Analysis, vol. 74, no. 1, pp. 160-197, 1987.

[13] M. Grillakis, J. Shatah, and W. Strauss, "Stability theory of solitary waves in the presence of symmetry, II," Journal of Functional Analysis, vol. 94, no. 2, pp. 308-348, 1990.

[14] E. W. Laedke and K. H. Spatschek, "Stability theorem for KdVtype equations," Journal of Plasma Physics, vol. 32, no. 2, pp. 263$272,1984$.

[15] T. B. Benjamin, “The stability of solitary waves," Proceedings of the Royal Society A Mathematical, Physical and Engineering Sciences, vol. 328, pp. 153-183, 1972.

[16] J. Bona, "On the stability theory of solitary waves," Proceedings of the Royal Society A Mathematical, Physical and Engineering Sciences, vol. 344, no. 1638, pp. 363-374, 1975.

[17] R. L. Pego and M. I. Weinstein, "Eigenvalues, and instabilities of solitary waves," Philosophical Transactions of the Royal Society of London. Series A. Mathematical, Physical Sciences and Engineering, vol. 340, no. 1656, pp. 47-94, 1992.

[18] M. I. Weinstein, "Lyapunov stability of ground states of nonlinear dispersive evolution equations," Communications on Pure and Applied Mathematics, vol. 39, no. 1, pp. 51-67, 1986.

[19] M. I. Weinstein, "Existence and dynamic stability of solitary wave solutions of equations arising in long wave propagation," Communications in Partial Differential Equations, vol. 12, no. 10, pp. 1133-1173, 1987.

[20] J. L. Bona, P. E. Souganidis, and W. A. Strauss, "Stability and instability of solitary waves of Korteweg-de Vries type," Proceedings of the Royal Society A Mathematical, Physical and Engineering Sciences, vol. 411, no. 1841, pp. 395-412, 1987.

[21] V. I. Karpman, "Stabilization of soliton instabilities by higher order dispersion: KdV-type equations," Physics Letters A, vol. 210, no. 1-2, pp. 77-84, 1996.

[22] V. I. Karpman, "Lyapunov approach to the soliton stability in highly dispersive systems. II. KdV-type equations," Physics Letters A, vol. 215, no. 5-6, pp. 257-259, 1996.

[23] W. Zhang, Q. Chang, and E. Fan, "Methods of judging shape of solitary wave and solution formulae for some evolution equations with nonlinear terms of high order," Journal of Mathematical Analysis and Applications, vol. 287, no. 1, pp. 1-18, 2003.

[24] T. Kato, “On the korteweg-de vries equation," Manuscripta Mathematica, vol. 28, no. 1-3, pp. 89-99, 1979.

[25] P. Hartman, Ordinary Differential Equations, Birkhauder, Boston, Mass, USA, 2nd edition, 1982.

[26] M. Reed and B. Simon, Methods of Mathematical Physics, vol. IV, Academic Press Inc, 2nd edition, 1978. 


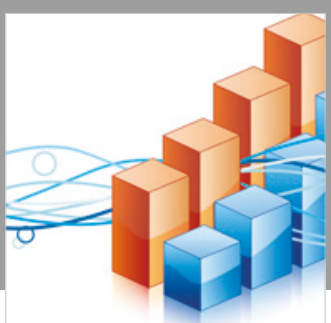

Advances in

Operations Research

\section{-n-m}
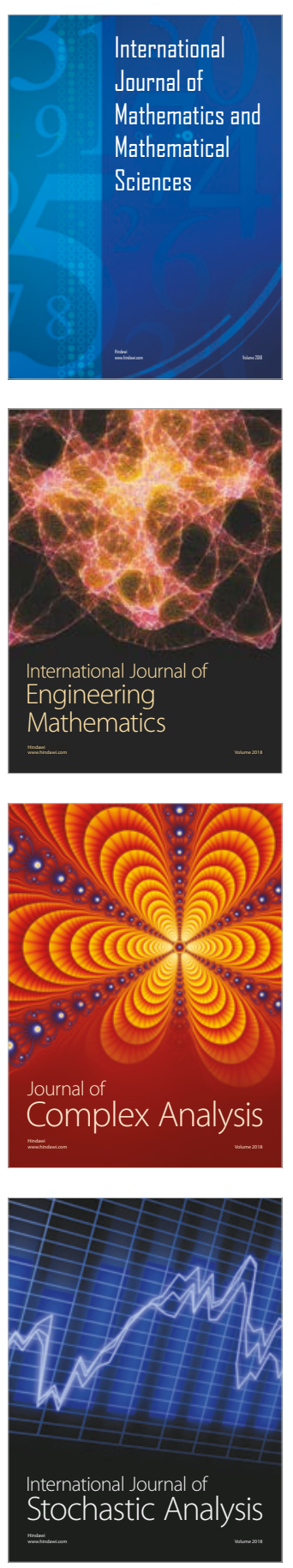
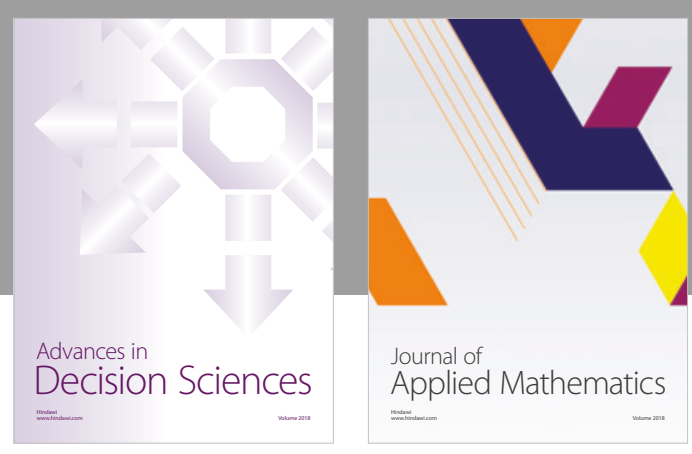

Journal of

Applied Mathematics
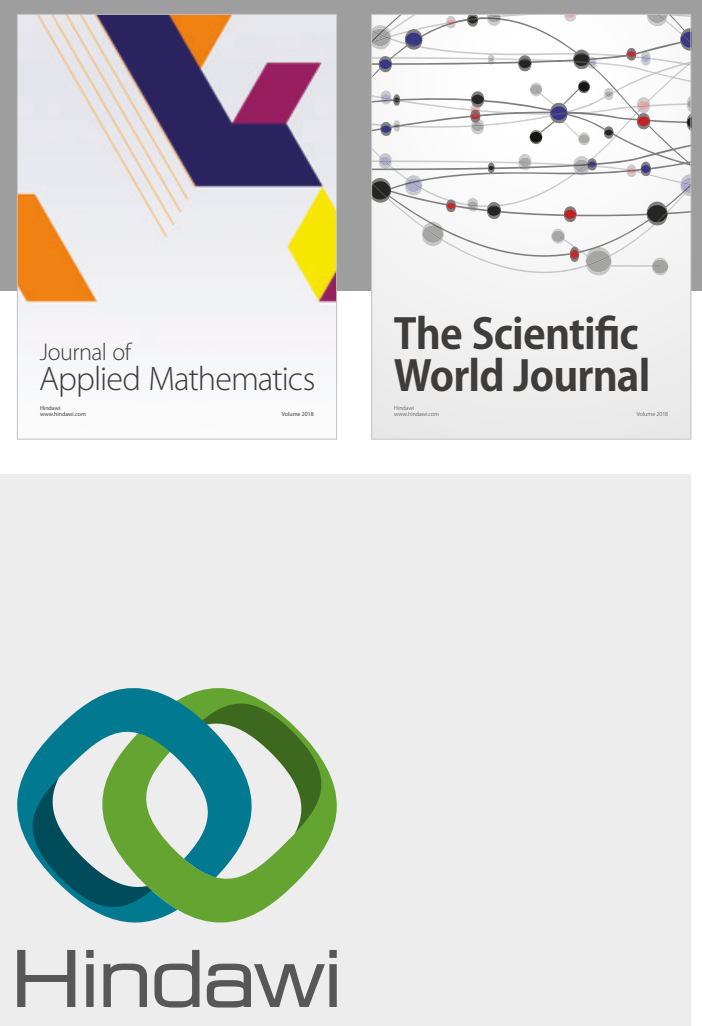

Submit your manuscripts at

www.hindawi.com

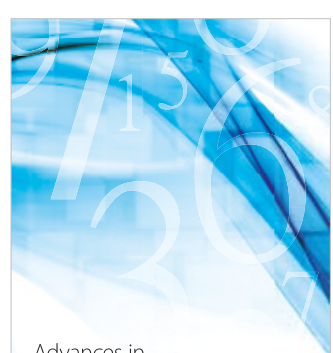

Advances in
Numerical Analysis
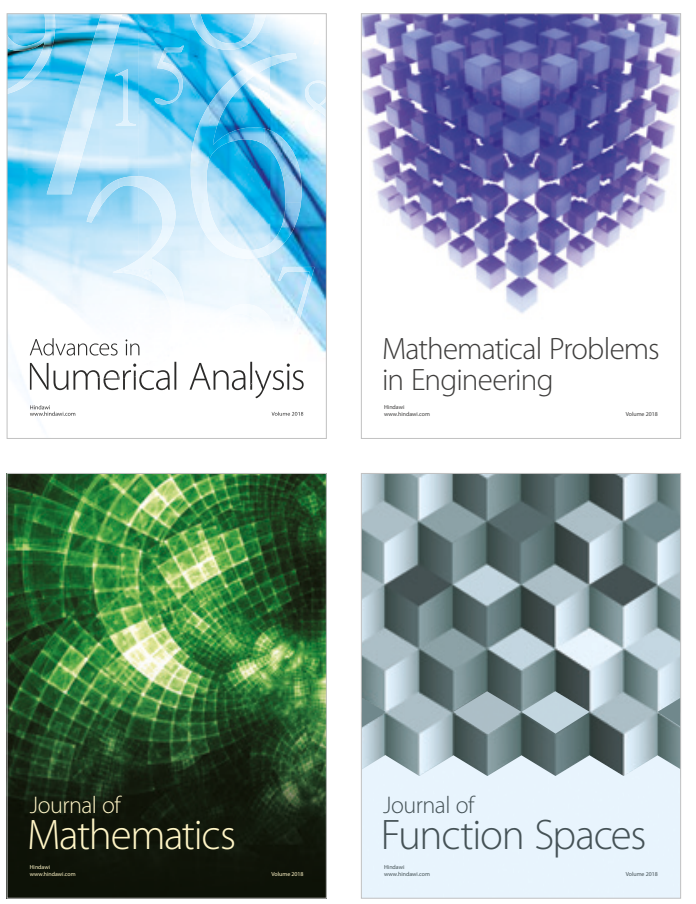

Mathematical Problems in Engineering

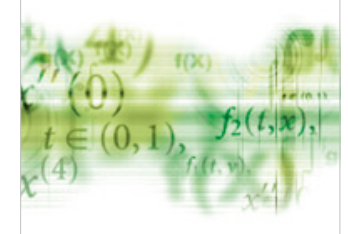

International Journal of

Differential Equations

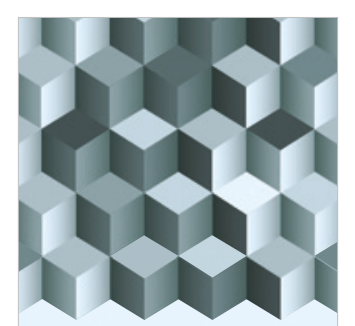

Journal of

Function Spaces
The Scientific

World Journal

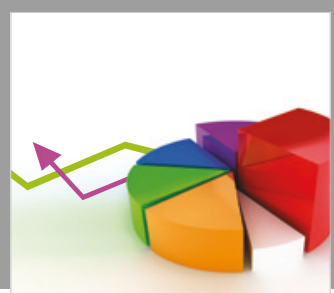

Journal of

Probability and Statistics
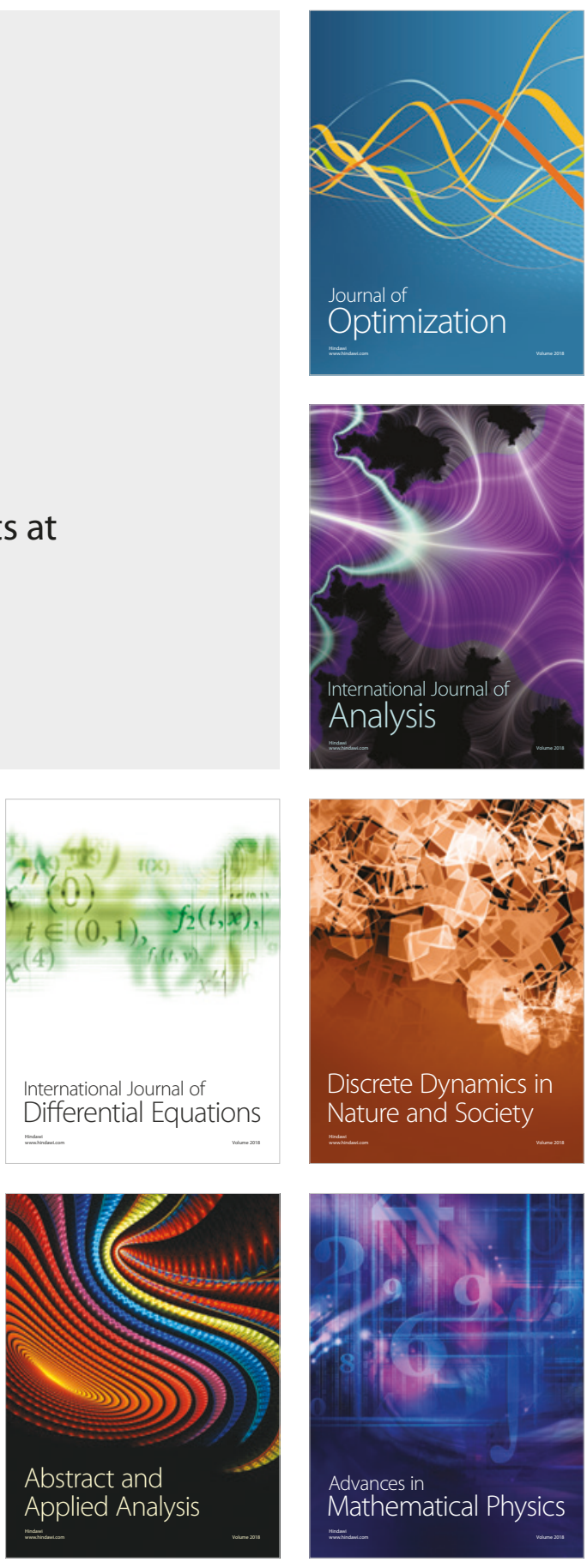